\title{
TREAT Fuel Motion Summary Report - SETH A-E Experiments
}

\author{
L. Ocampo \\ J. Johnson \\ S. Thompson \\ J. Hix \\ S. Watson \\ D. Chichester
}

September 2019

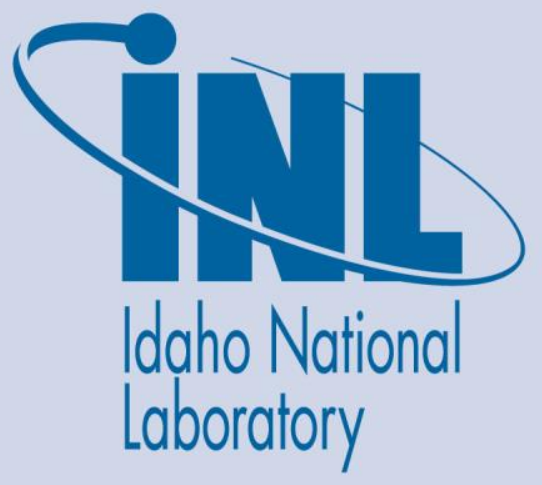

INL is a U.S. Department of Energy National Laboratory operated by Battelle Energy Alliance 


\section{DISCLAIMER}

This information was prepared as an account of work sponsored by an agency of the U.S. Government. Neither the U.S. Government nor any agency thereof, nor any of their employees, makes any warranty, expressed or implied, or assumes any legal liability or responsibility for the accuracy, completeness, or usefulness, of any information, apparatus, product, or process disclosed, or represents that its use would not infringe privately owned rights. References herein to any specific commercial product, process, or service by trade name, trade mark, manufacturer, or otherwise, does not necessarily constitute or imply its endorsement, recommendation, or favoring by the U.S. Government or any agency thereof. The views and opinions of authors expressed herein do not necessarily state or reflect those of the U.S. Government or any agency thereof. 


\title{
TREAT Fuel Motion Summary Report - SETH A-E Experiments
}

\author{
L. Ocampo \\ J. Johnson \\ S. Thompson \\ J. Hix \\ S. Watson \\ D. Chichester
}

September 2019

\author{
Idaho National Laboratory \\ Nuclear Nonproliferation Division \\ Radiochemistry \& Nuclear Measurements \\ Idaho Falls, Idaho 83415
}

http://www.inl.gov

Prepared for the

U.S. Department of Energy

Office of Nuclear Energy

Under DOE Idaho Operations Office

Contract DE-AC07-05ID14517 



\section{Radiochemistry and Nuclear Measurements}

\section{TREAT Fuel Motion Summary Report - SETH A-E Experiments}

INL/EXT-19-55235

September 2019
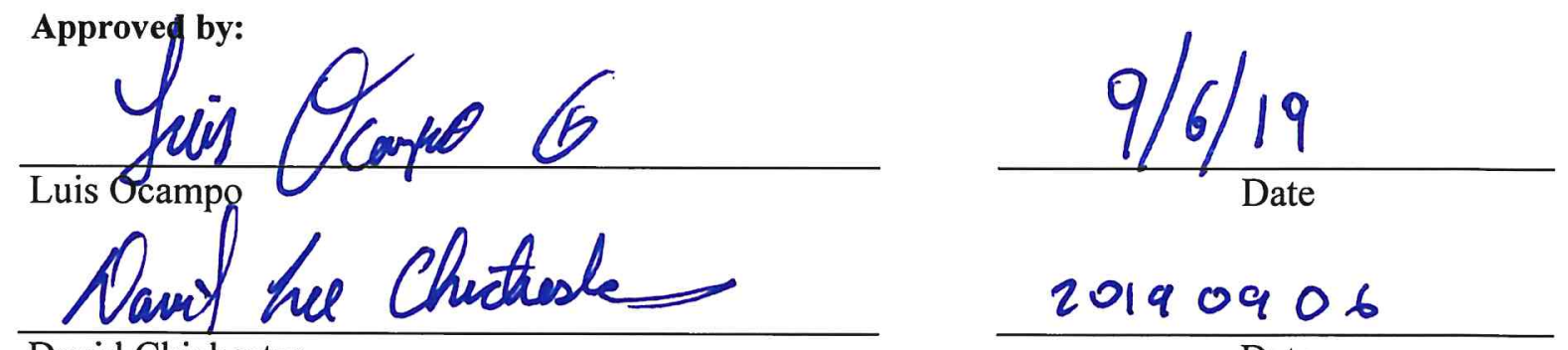

David Chichester

20190906 Date 



\section{EXECUTIVE SUMMARY}

In support of the Accident Tolerant Fuels program, experiment devices are being developed to enable transient testing of pressurized water reactor (PWR) type fuel specimens. In fiscal year 2019 several fueled tests were conducted in the Transient Reactor Test Facility (TREAT) using the Separate Effects Test Holder (SETH). Five different SETH capsules (SETH A-E) were assembled, each holding a 10-pellet rodlet with $4.9 \%$ enriched, commercially-produced fresh $\mathrm{UO}_{2}$ pellets in zirconium alloy cladding with pellet dimensions having typical PWR radial dimensions and a total pellet stack length measuring $10.16 \mathrm{~cm}$. The rodlet's length allows the specimen to be axially positioned in the center of the core with minimal flux variation over the height of the rodlet. The fuel motion monitoring system (FMMS) is located on the north side of the TREAT reactor. Having direct line-of-sight of the core enables the FMMS to collect spatially-resolved data for fast neutrons generated due to fission occurring in experiment specimens. The FMMS contains 360 channels that make up a 10 (horizontal) by 36 (vertical) array of viewing slots; for the SETH A-E test series 96 proton-recoil scintillator (PRS) detectors were installed in the system. The fast neutrons are detected in the PRS detectors from light produced by the recoil of protons interacting with $\mathrm{ZnS}(\mathrm{Ag})$ grains in each detector's scintillator button.

This report focuses on the FMMS data captured in the SETH A-E experiments, with multiple transients varying in reactivity addition and length. The first three experiments had a total reactor energy of $101 \mathrm{MJ}$ each and were critical in understanding the FMMS field of view, lead shielding, and other TREAT data acquisition systems. SETH-B2 and C had a reactivity addition of almost double the previous transients and served as a demonstration of the detector array behavior under increased reactor energy. The final two SETH experiments (D and E) were aimed at reaching cladding melting temperatures with a reactor energy over $500 \mathrm{MJ}$ for each test. In preparation for the higher energy transients, the FMMS lead shielding was reduced to 0.5 inches from the 6 inches used in SETH A-C. This was expected to saturate the detectors at the peak of the transient in exchange for higher sensitivity at lower energies after the peak, when the motion was expected. SETH-D was clipped at approximately 3 seconds, which was too early for the FMMS to capture any rodlet motion. SETH-E had the same reactivity addition as SETH-D but the transient time was extended to 18 seconds. The FMMS captured the downward motion of the rodlet in SETH-E as the count rate significantly changed for the detectors viewing the specimen. Snapshots taken throughout the transient helped identify the approximate time at which the motion initiated. Further analysis of the individual detector data captured on a per-millisecond time basis resulted in a determination that the approximate time for the onset of the downward motion was $2.84 \pm 0.02$ seconds. Neutron radiographs confirmed that downward motion occurred for SETH-D and SETHE. Motion in SETH-D could also have been captured by the FMMS if the transient time had been longer for SETH-D. Ultimately, these final two tests served to demonstrate the FMMS's ability to track fuel motion during transient experiments at TREAT. 


\section{CONTENTS}

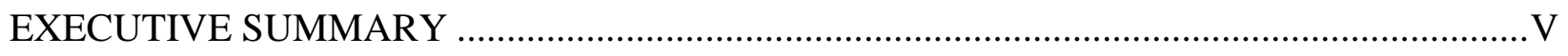

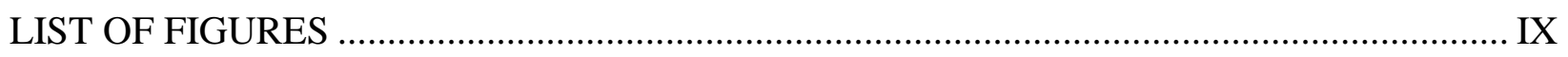

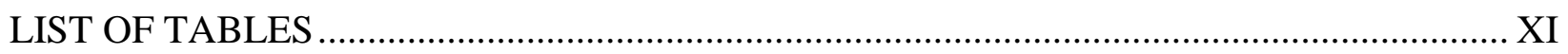

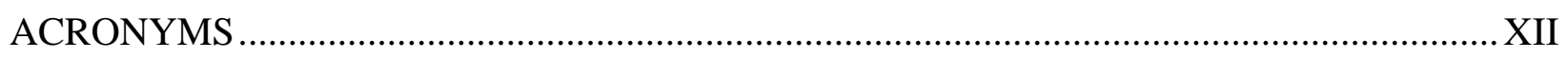

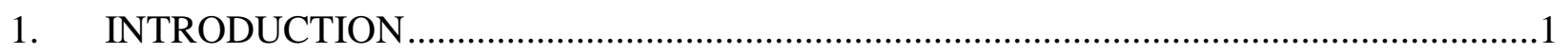

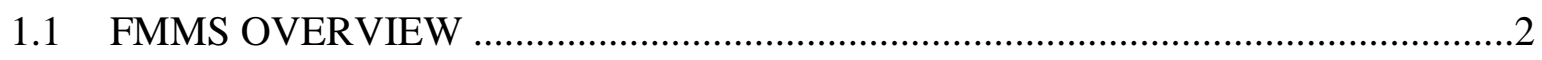

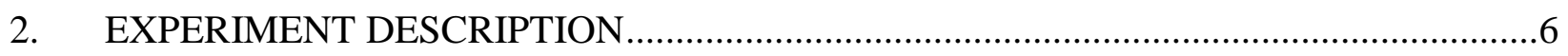

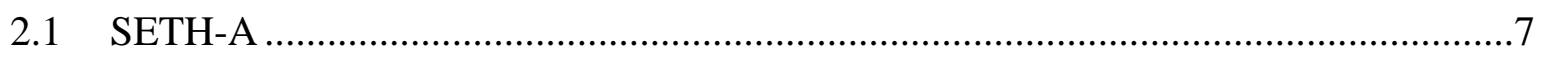

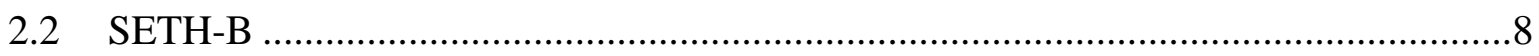

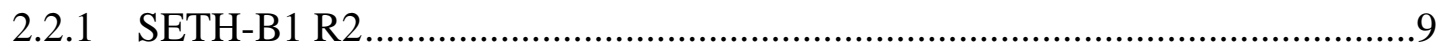

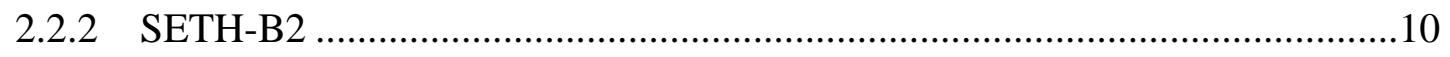

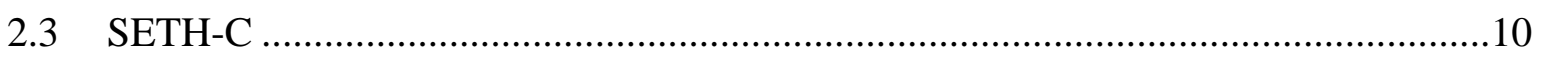

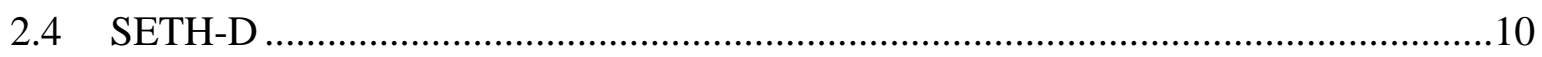

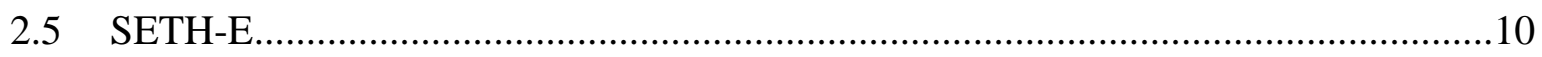

3. FUEL MOTION MONITORING SYSTEM RESULTS .................................................11

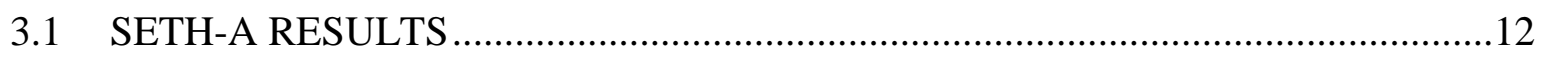

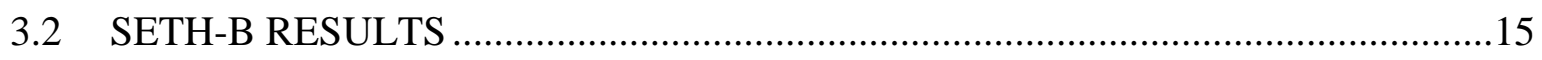

3.2.1 SETH-B1 R2 RESULTS .................................................................

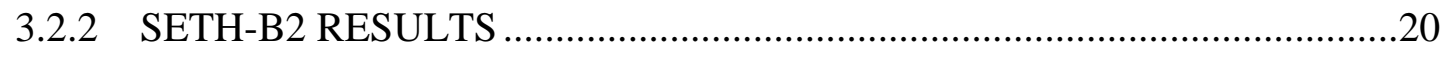

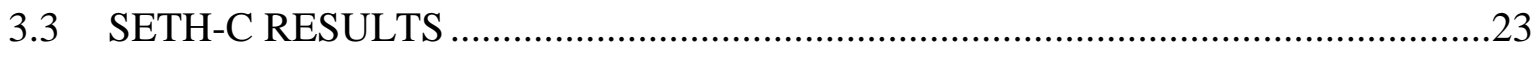

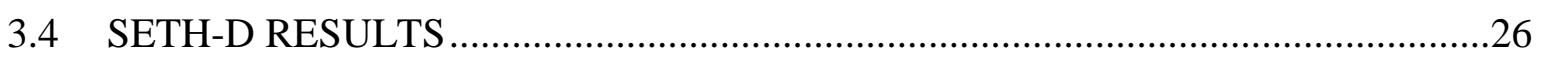

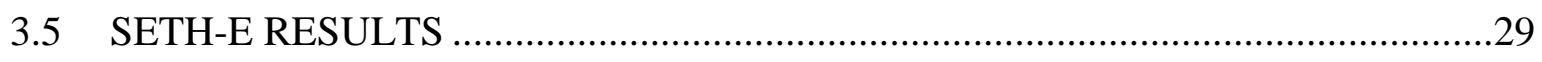

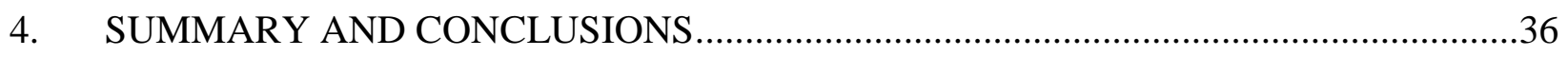

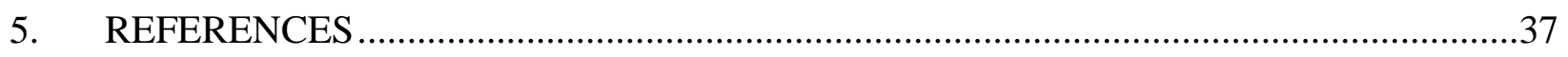




\section{LIST OF FIGURES}

Figure 1. Overview of TREAT features, including the FMMS located in the north side [1].

Figure 2. Photograph of the FMMS detector cabinet ..................................................... 4

Figure 3. FMMS detector array with control panel and associated equipment. ......................... 5

Figure 4. Diagrams and photographs of the SETH module and BUSTER irradiation

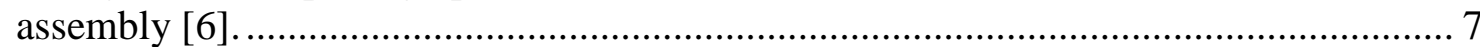

Figure 5: SETH-A Photo (left, one half of insulation removed) and TC Locations from Bottom End Cap, Dimensions in cm (right) [3] .................................................. 8

Figure 6: Images of SETH-B through -E Specimen, Instrument, and Assembly Photograph (left) and Design Rendering (right) [3].

Figure 7. SETH-A FMMS neutron sum intensity plot and vertical profile for the central columns.

Figure 8. Profiles of selected FMMS detector count rates (counts per $1 \mathrm{~s}$ ) and reactor power over the duration of the SETH-A transient.

Figure 9. Snapshots of the FMMS during SETH-A at different times throughout the transient.

Figure 10. SETH-B FMMS neutron sum intensity plot and vertical profile for the main columns.

Figure 11. Profiles of selected FMMS detectors and reactor power over the duration of the SETH-B transient.

Figure 12. Snapshots of the FMMS during SETH-B at different times throughout the transient.

Figure 13. SETH-B1 R2 FMMS neutron sum intensity plot and central columns vertical profile.

Figure 14. Profiles of selected FMMS detectors and reactor power over the duration of SETH-B1 R2.

Figure 15. Snapshots of the FMMS during SETH-B1 R2 at different times.

Figure 16. SETH-B2 FMMS neutron sum intensity plot and central columns vertical profile.

Figure 17. Profiles of selected FMMS detectors and reactor power over the duration of the SETH-B2 transient.

Figure 18. Snapshots of the FMMS during SETH-B2 at different times throughout the transient.

Figure 19. SETH-C FMMS neutron sum intensity plot and central columns vertical profile.

Figure 20. Profiles of selected FMMS detectors and reactor power over the duration of the SETH-C transient. 
Figure 21. Snapshots of the FMMS during SETH-C at different times throughout the transient.

Figure 22. SETH-D FMMS neutron sum intensity plot and central columns vertical profile.

Figure 23. Profiles of selected FMMS detectors and reactor power over the duration of the SETH-D transient.

Figure 24. Snapshots of the FMMS during SETH-D at different times throughout the transient. Detector saturation was observed between 1.1 and 1.5 seconds (B). 28

Figure 25. SETH-E FMMS neutron sum intensity plot and vertical profile for the central columns.

Figure 26. Profiles of selected FMMS detectors and reactor power over the duration of SETH-E. The zoomed box highlights the detector profiles at 3 seconds. 30

Figure 27. Enhancement of Figure 26, for time between 2 and 4 seconds, illustrating the observed downward motion of fuel in SETH-E.

Figure 28. Snapshots of the FMMS during SETH-E at different times throughout the transient identified as A through $\mathrm{D}$. The downward motion can be seen between $\mathrm{C}$ and $\mathrm{D}$

Figure 29. Snapshots of the FMMS during SETH-E at times before and after the movement of the rodlet. Detectors 165, 175, 185 and 195 are identified in Snapshot A. The downward motion is highlighted between Snapshots B and C with white circles.

Figure 30. Millisecond data for detectors 165 and 195. The solid lines represent smoothed fits to the data using a Savitzky-Golay filter.

Figure 31. Filtered detector data for detectors 165 and 195. The dashed blue line identifies the approximate time when the downward motion started. 34

Figure 32. Post transient neutron radiographs for higher energy SETH tests [3]. 35 


\section{LIST OF TABLES}

Table 1. SETH transient parameters .......................................................................... 11 


\section{ACRONYMS}

BUSTER Broad Use Specimen Transient Experiment Rig

ECF Energy Coupling Factor

FMMS Fuel Motion Monitoring System

MARCH Minimal Activation Retrievable Capsule Holder

PWR Pressurized Water Reactor

PRS Proton Recoil Scintillator

SETH Separate Effects Test Holder

TC Thermocouple

TCF Transient Correction Factor

TREAT Transient Reactor Test Facility 


\section{TREAT Fuel Motion Summary Report - SETH A-E Experiments}

\section{INTRODUCTION}

The Transient Reactor Test Facility (TREAT) is used to study fuel melting behavior, interactions between fuel and coolant, and the potential for propagation of failure to adjacent fuel pins under conditions ranging from mild upsets to severe accidents. TREAT is an air-cooled reactor driven by a core of graphite blocks having a small concentration of dispersed uranium oxide. Columns of these graphite-fuel blocks are hermetically encapsulated in zirconium alloy sheet metal canisters. Aluminum-sheathed unfueled graphite blocks are attached to the top and bottom of each fuel column; forming a discrete fuel assembly with $1.2 \mathrm{~m}$ of active core length. Along with control rod, experiment, and graphite reflector assemblies, these fuel assemblies are placed on a $19 \times 19$ gridplate with 361 available positions; creating a configurable core that can be adjusted to suit particular nuclear parameters or experimental objectives. A few fuel assemblies are typically removed from the central core positions to create a cavity for experiments. Experiment assemblies are typically removed from or placed into the core through a slot in the reactor's upper rotating shield plug, handled outside the reactor using shielded casks, and stowed below grade in storage holes when not in use. Four beam-port slots can be opened through the vertical concrete shield walls and permanent graphite reflector surrounding the above-grade core, one on each of the four walls, to provide various measurement capabilities. Two beam ports are currently in use, one for a neutron radiography beam and the other for a fuel motion monitoring system (FMMS), sometimes referred to as a hodoscope. The FMMS is capable of collecting spatially resolved data for fast neutrons born in experiment specimens [1].

TREAT experiment devices are being developed to enable transient testing of Light Water Reactor type fuel specimens, including fuel concepts with enhanced accident tolerance as well as other types of compatible specimens. While many tests and their supporting irradiation device designs focus on creating prototypic boundary conditions for integral scale testing, there is also a great need for cost-effective science-based and separate-effects scale tests. These separate-effects tests often support phenomena identification for more focused experimentation in subsequent integral scale testing. An approach to this type of testing arranges fresh fuel specimens in low activation hardware so that they can be easily extracted and shipped for Post Irradiation Examination within weeks. This approach is possible due to the relatively minor radioactivity accumulated during brief TREAT irradiations. The Minimal Activation Retrievable Capsule Holder (MARCH) irradiation device includes multipurpose modules that can be configured in various ways. The heart of the design is a reusable stainless-steel containment structure, termed the Broad Use Specimen Transient Experiment Rig (BUSTER), which constitutes the reactor core thermal/mechanical interface and serves as the credited safety containment [2]. One of the modules designed for use in the MARCH system is the Separate Effects Test Holder (SETH). SETH is designed to support a 10-pellet rodlet of $4.9 \%$ enriched, commercially-produced fresh $\mathrm{UO}_{2}$ pellets in zirconium alloy cladding having typical PWR radial dimensions and a total pellet stack length measuring 10.16 $\mathrm{cm}$. Five of these capsules were constructed and identified as SETH-A through -E, each containing 
1-atmosphere helium. SETH-B through -E also held four 1-mm diameter titanium-sheath type K thermocouples (TC) welded to the specimen cladding surface and a fiber-optic pyrometer [3].

\subsection{FMMS Overview}

The FMMS is used to image the internals of an experiment enclosed in an opaque test train placed in the center of the TREAT reactor during reactor power excursion transients, see Figure 1 . There are two collimators associated with the FMMS. The front collimator is a horizontal slit with an aperture $38.1-\mathrm{mm}$ tall by $215.6-\mathrm{mm}$ wide that provides general shielding of the rear collimator. The rear collimator is a stack of steel plates with 360 milled, angled slots. The rear collimator projects line-of-sight neutrons from the center of the reactor's core to an array of proton-recoil scintillator (PRS) fast-neutron detectors. The FMMS features 360 channels that make up a 10 by 36 detector array (see Figure 2). For the SETH-A through -E test series 96 PRS detector assemblies were installed in the FMMS. Each PRS detector assembly consists of PRS buttons matched to photomultiplier tubes (PMTs), one on each channel slot. A PRS button consists of a thin wafer made from a blend of $5 \% \mathrm{ZnS}(\mathrm{Ag})$ grains dispersed in a $95 \%$ Lucite medium, the wafer has dimensions of $2.78 \mathrm{~mm} \times 12.26 \mathrm{~mm} \times 15.90 \mathrm{~mm}$. The $\mathrm{ZnS}(\mathrm{Ag}) / \mathrm{Lucite}$ wafer is then adhered to two half-cylindrical Lucite waveguides using optical cement, forming a PRS button. Each PRS button is coupled to a PMT with optical gel, yielding a PRS detector assembly. The fast neutrons are detected from light produced by the recoil of protons interacting with the $\mathrm{ZnS}(\mathrm{Ag})$ grains [4]. The currently-installed 96 channels are spread between four columns. The two centermost columns have 36 channels and the neighboring side columns have 12 channels, centered in each column.

The FMMS motion control system has both local and remote-control operator interfaces. The local control interface consists of a laptop which can be used to move the FMMS platform while near the FMMS, for troubleshooting and maintenance. The remote interface is part of the data acquisition system, which integrates control of the FMMS platform position with data collection by sending commands to the motion control system. The data acquisition system may be controlled by a local operator near the reactor or remotely from the TREAT control building (Building 724). The remote and local control interfaces communicate with the motion control system control panel located in the FMMS control room. The FMMS control panel contains a National Instruments processor, and the associated equipment required to control the stepper motors, servo motors, limit switches, and position feedback devices required to position the FMMS, see Figure 3. There are two separate motion systems, the source positioner and the detector platform. The Source Positioner is used to characterize the detectors which make up the detector array located at the north end of the FMMS. The detector array is characterized by positioning a radioactive source in front of the individual detectors in the array and recording their response. The motion control system uses stepper motors to position the radiation source in the $\mathrm{X} / \mathrm{Y}$ plane. The detector platform positions the rear collimator and detector array as required to appropriately image experiments in the reactor. The motion control system uses a stepper motor to adjust the horizontal position, and two servo motors to adjust the vertical position of the platform [5]. 


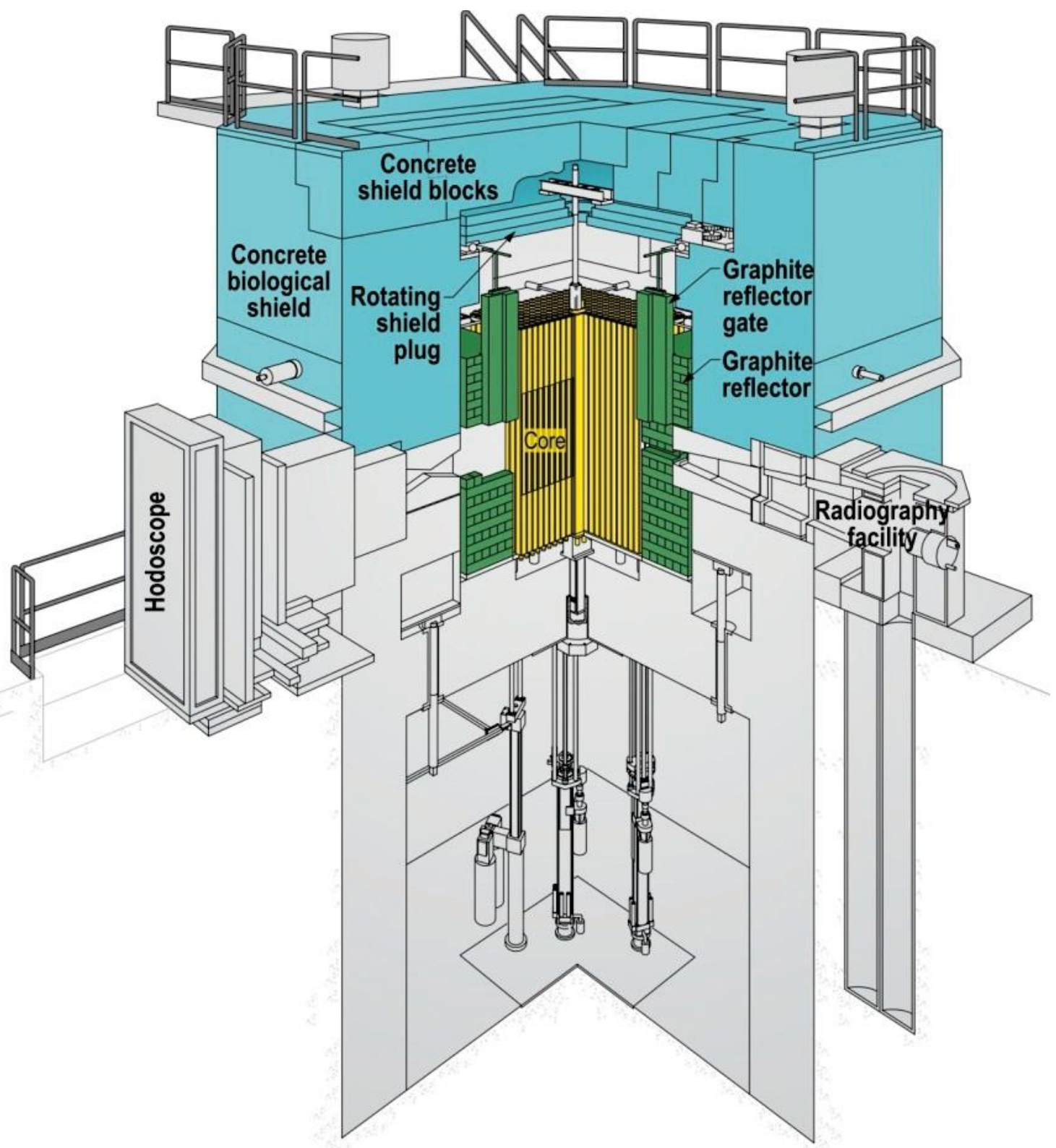

Figure 1. Overview of TREAT features, including the FMMS located in the north side [1]. 


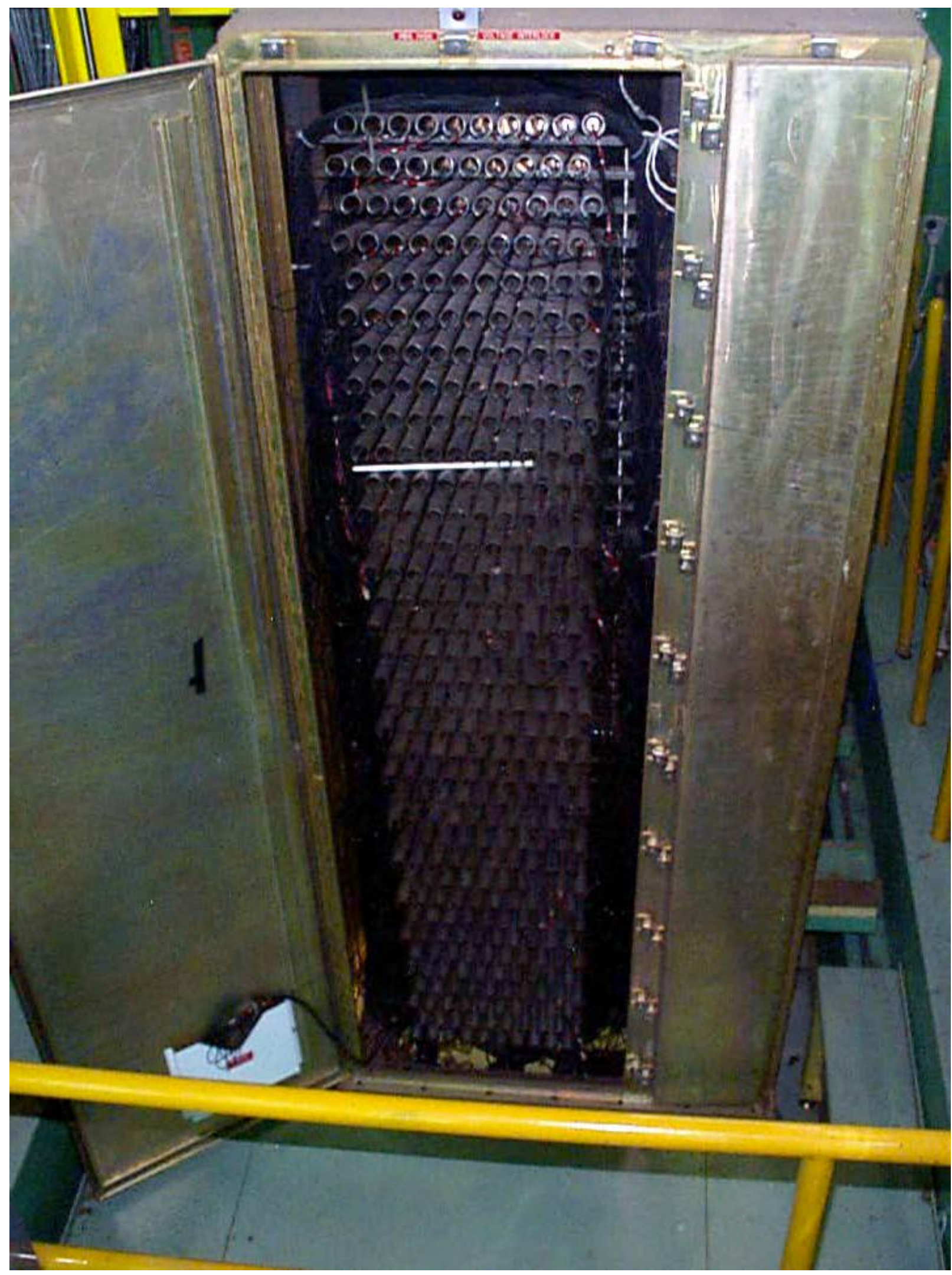

Figure 2. Photograph of the FMMS detector cabinet 


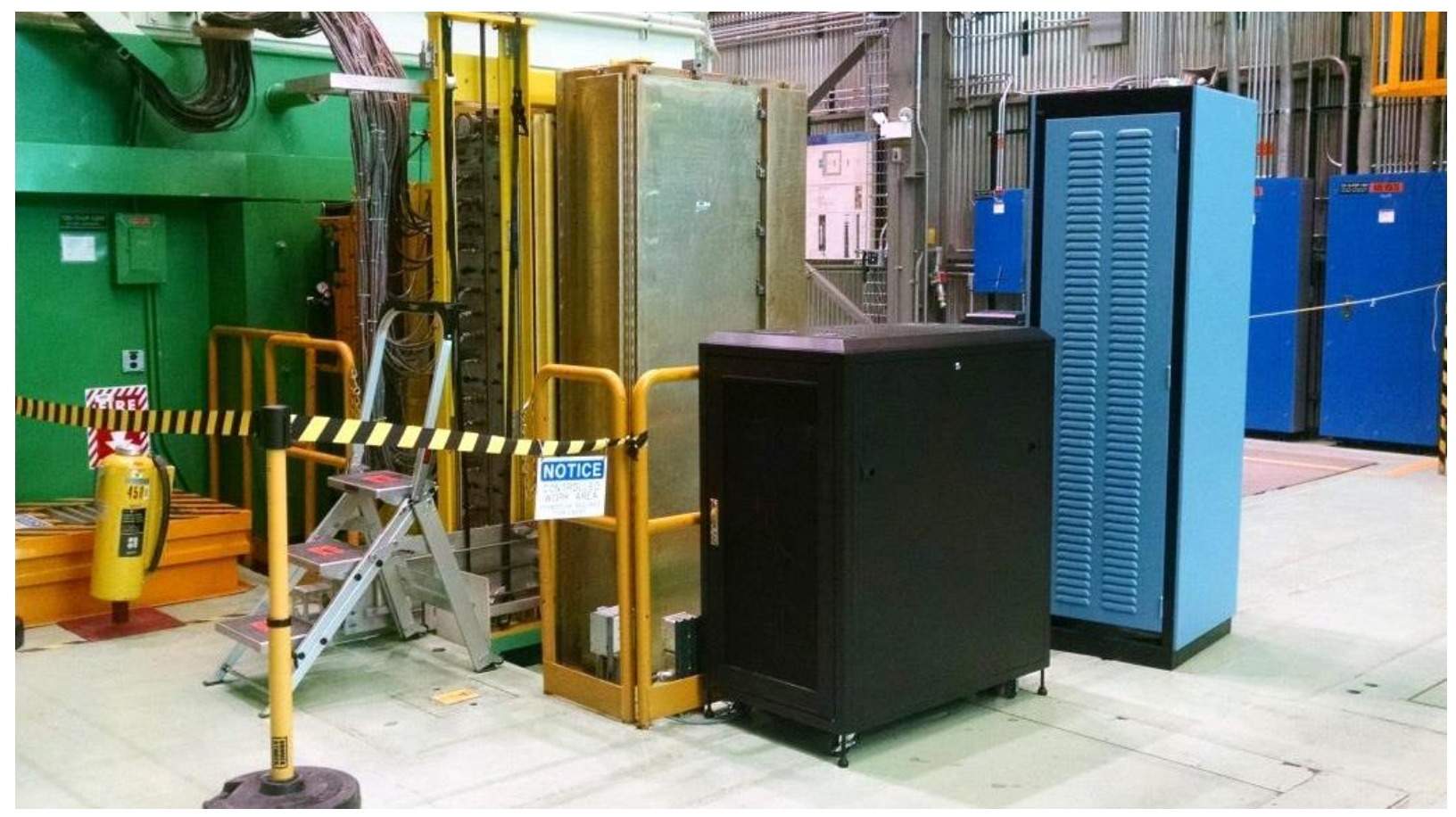

Figure 3. FMMS detector array with control panel and associated equipment. 


\section{EXPERIMENT DESCRIPTION}

Fuel-bearing transient irradiation experiments were performed in order to support fuel safety research for the US Department of Energy Office of Nuclear Energy's Accident Tolerant Fuels program. Historically, the most common practice for determining transient correction factors (TCFs) could be inferred by comparing steady state and transient fission wire irradiations. The product of these factors and the core's transient power shape were needed to predict safety-case performance of high-energy final transient tests. Schedule implications of dosimeter evaluation logistics and the scarcity of historic fission wire materials, which were typically uncommon alloys having very dilute uranium concentration to prevent their melting during transients, required an alternate approach for this test series using in-situ calorimetric methods. In the SETH calorimetry approach, temperature measurements of the rodlet's cladding surfaces were correlated with thermal models of the experiment [3].

The SETH experiments used the previously mentioned SETH capsules, shown in Figure 4. All of the capsules were filled with helium gas at room temperature and roughly atmospheric pressure pre-transient condition. Zirconium alloy end caps and cladding encapsulated the pellet stack. Each SETH capsule hangs from a large hanger rod inside the MARCH-BUSTER configuration. The rod's length allows the specimen to be axially positioned in the center of the core. The first of these capsules (SETH-A) was unique with thermal insulation surrounding the rodlet. The SETH-B through -E rodlets were not surrounded by thermal insulation but were instead encompassed by an aluminum cage designed to support instrumentation. These four capsules were outfitted with infrared multispectral pyrometry to determine whether this new technology was suited to providing fast-response, non-contact temperature measurements in a transient nuclear environment. 


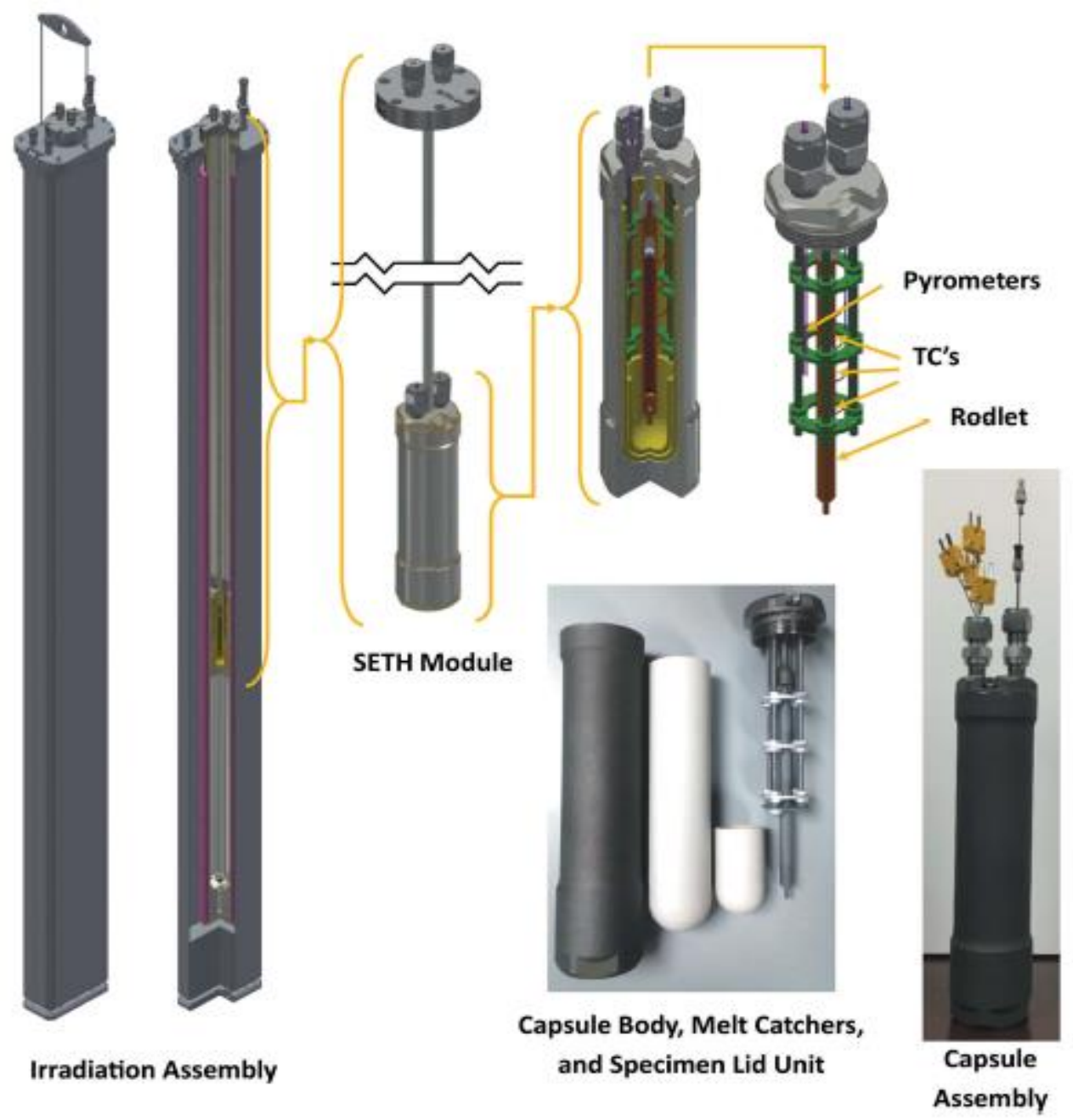

Figure 4. Diagrams and photographs of the SETH module and BUSTER irradiation assembly [6].

\subsection{SETH-A}

SETH-A was an experiment used for developing the method for energy coupling factor (ECF) calorimetry, in order to determine coupling factors for the remaining SETH based irradiations. SETH-A was subjected to a $0.6 \% \Delta \mathrm{k} / \mathrm{k}$ reactivity addition to determine the ECF. This was transient number 2913. In order to enable the ECF calorimetry approach modifications were made for the SETH-A capsule. The TCs were oriented so that the sheath tip and junction area were normal to the cladding surface and welded using an autogenous micro tungsten arc weld to minimize thermal mass, see Figure 5. A blank Conax ${ }^{\circledR}$ sealant was used to plug the vacant gland body. The rodlet was surrounded by neutronically transparent alumina/silica-based microporous insulation (MICROSIL by Zircar®). The insulator's main purpose was to enable high accuracy calorimetry by giving a configuration where heat losses from the rodlet were predominantly conductive rather than radiative as they would be in their absence. In this way, low energy transients can be performed to cause a measurable rodlet temperature increase in a few seconds, but well within the 
temperature measurement range for the TCs. Out-of-core, electrically heated mockup testing and thermal modeling were used to correct for heat losses prior to the TCs measuring peak temperature for higher accuracy ECF determination [7].
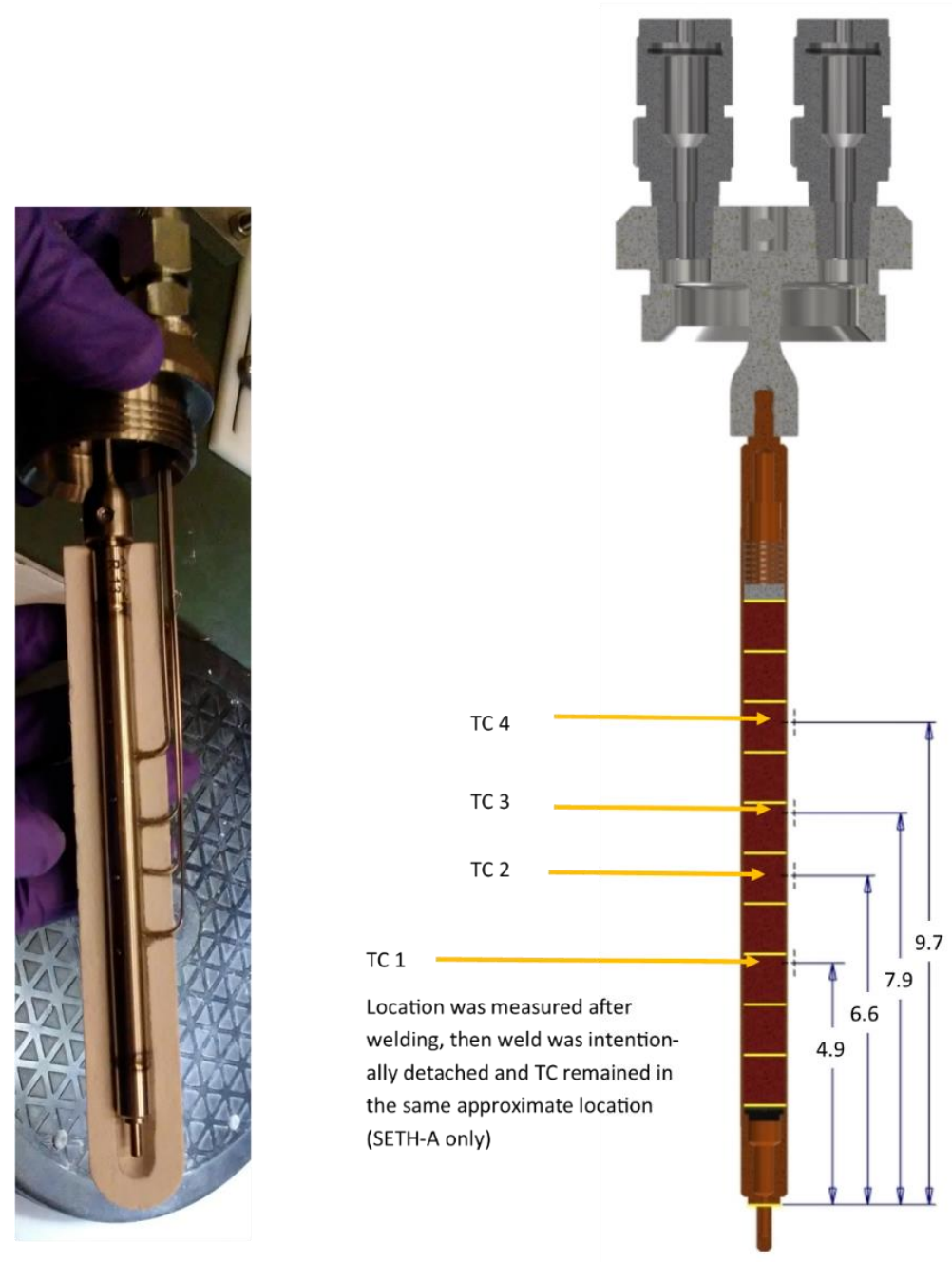

Figure 5: SETH-A Photo (left, one half of insulation removed) and TC Locations from Bottom End Cap, Dimensions in cm (right) [3].

\subsection{SETH-B}

SETH-B was used for three separate experiment tests using the same hardware. The first test, SETH-B1, subjected the capsule to a $0.6 \% \Delta \mathrm{k} / \mathrm{k}$ reactivity addition, corresponding to $100 \mathrm{MJ}$ of core energy. This was transient number 2914. The TCs in capsules SETH-B through -E were placed at the same nominal axial locations as SETH-A. TC1 and TC3 were the same diamondpacked type used in SETH-A while TC2 and TC4 were nearly identical, except that their junctions were grounded to compare both their response time and sensitivity to noise in a newly constructed data acquisition system at TREAT. The aluminum cage supported two silica optical fibers which 
were cleaved at an angle, polished, and aligned so that they were focused on the same area of the cladding surface, on the opposite side of the line of TCs, at approximately the axial center of the rodlet's fueled region. Both fibers were connected to multispectral pyrometers at TREAT to account for surface emissivity effects in real time. An image of the SETH-B through -E rodlet/instrumentation package can be seen in Figure 6 [3].
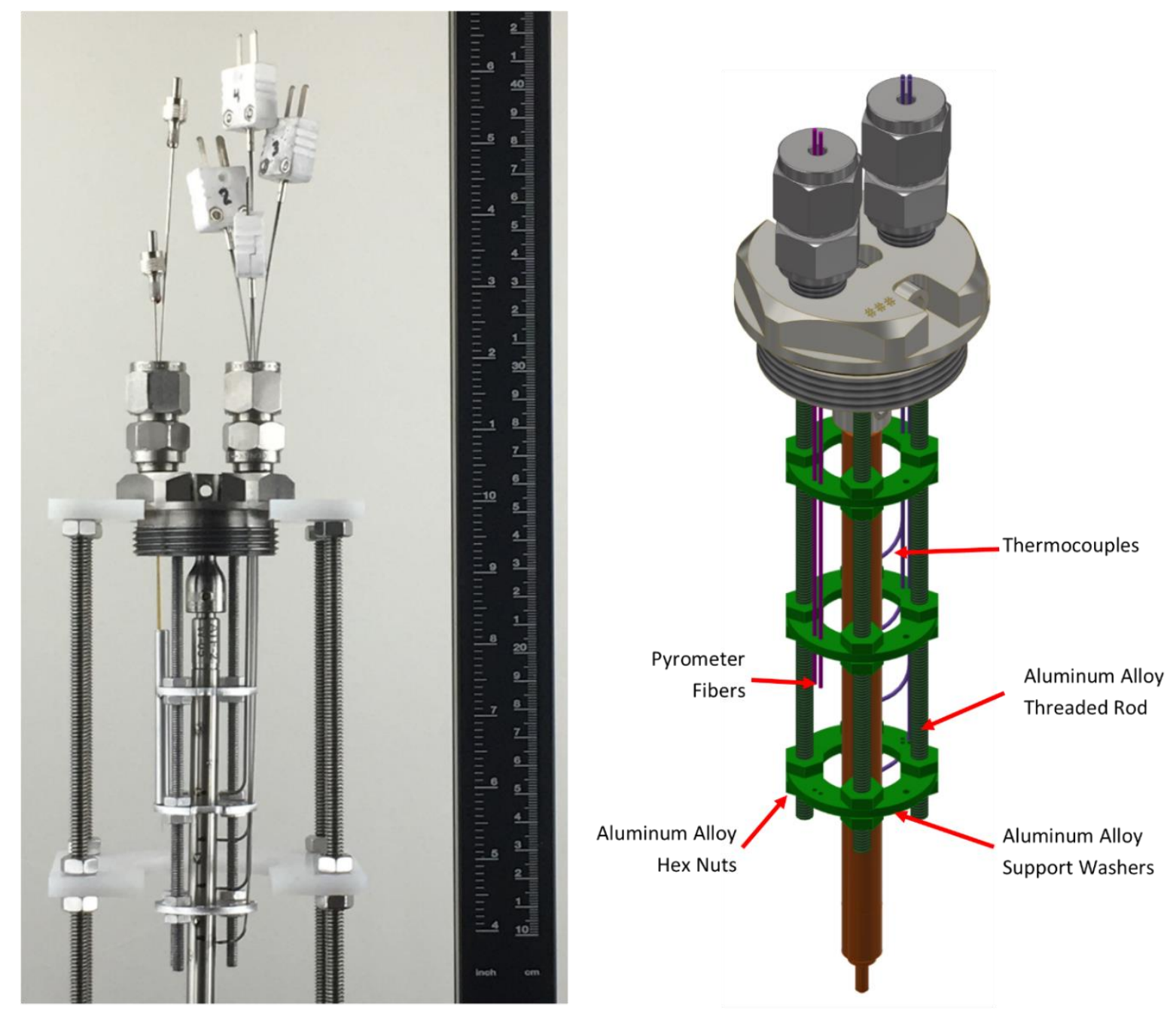

Figure 6: Images of SETH-B through -E Specimen, Instrument, and Assembly Photograph (left) and Design Rendering (right) [3].

\subsubsection{SETH-B1 R2}

The SETH-B1 transient was repeated with the same $0.6 \% \Delta \mathrm{k} / \mathrm{k}$ reactivity addition to investigate different pyrometry data acquisition settings. This was transient number 2915 , the small reactivity step insertion yielded a relatively slow pulse with low energy release but it was adequate to cause a measurable temperature rise in the specimen 


\subsubsection{SETH-B2}

The secondary experiment was SETH-B2 and it was subjected to a $1.15 \% \Delta \mathrm{k} / \mathrm{k}$ reactivity addition to produce $143 \mathrm{MJ}$ of core energy. This was transient number 2917, it was clipped to yield moderate energy releases where instrumentation was expected to survive.

\subsection{SETH-C}

SETH-C was transient number 2919 . It was subjected to a $1.15 \% \Delta \mathrm{k} / \mathrm{k}$ reactivity addition and the same step insertion as SETH-B2 but clipped much later to yield increased specimen temperatures.

\subsection{SETH-D}

SETH-D was subjected to $1.69 \% \Delta \mathrm{k} / \mathrm{k}$ reactivity addition, resulting in approximately $500 \mathrm{MJ}$ total reactor energy. This was done with the objective to barely achieve the zirconium's melting temperature of $1850{ }^{\circ} \mathrm{C}$ for partial cladding melt. The capsule hardware was identical to SETH-C.

\subsection{SETH-E}

SETH-E was irradiated with the same step insertion as SETH-D but clipped several seconds after the clipping time for SETH-D, to allow additional heating of the rodlet and so that a more intense fast neutron would be generated in the rodlet at later times, to allow it to be observed by the FMMS when the cladding deformed and it experienced gravity-assisted motion downward. The measured peak cladding temperatures was $2113^{\circ} \mathrm{C}$. 


\section{FUEL MOTION MONITORING SYSTEM RESULTS}

All 96 detectors were active and counting during all the SETH transients. The first three transients provided critical data to center the FMMS in order to position its view towards the center of the core where the specimens were located. This same data was used to calculate the number of lead shields that were needed to be placed in front of the FMMS to avoid early saturation of the detectors. The main parameters and the number of lead sheets used for all transients is listed in Table 1. The FMMS results are displayed in a graphical interface containing all the installed detectors in their respective location along with a vertical profile of the four columns being used. Since only 96 detectors were used during these experiments the rest of the interface is shown as black squares.

The first 5 transients (SETH-A through E) had the maximum amount of lead shielding in place resulting in a low count rate. Upon the removal of 11 sheets of lead, the count rate increased by an order of magnitude, showing an increased sensitivity. The last two transients (SETH-D \& SETHE) were important due to the higher reactivity step insertion, resulting in significantly higher temperatures and achieving the objectives of melting the cladding. This downward movement was seen by the FMMS during SETH-E as this transient was clipped approximately seven seconds after fuel motion. This same motion was not observed in the previous transient (SETH-D) as it was clipped at approximately 3 seconds, the approximate time when cladding melting occurred.

Table 1. SETH transient parameters

\begin{tabular}{|c|c|c|c|c|}
\hline $\begin{array}{l}\text { Transient } \\
\text { Number }\end{array}$ & Test ID & $\begin{array}{l}\text { Step Insertion } \\
\quad(\% \Delta K / \mathbf{k})\end{array}$ & $\begin{array}{l}\text { Number of FMMS } \\
\text { lead shielding sheets }\end{array}$ & $\begin{array}{l}\text { Total Reactor } \\
\text { Energy (MJ) }\end{array}$ \\
\hline 2913 & SETH-A & 0.6 & 12 & 101 \\
\hline 2914 & SETH-B & 0.6 & 12 & 101 \\
\hline 2915 & SETH-B1 R2 & 0.6 & 12 & 101 \\
\hline 2917 & SETH-B2 & 1.15 & 12 & 143 \\
\hline 2919 & SETH-C & 1.15 & 12 & 299 \\
\hline 2922 & SETH-D & 1.69 & 1 & 501 \\
\hline 2924 & SETH-E & 1.69 & 1 & 656 \\
\hline
\end{tabular}




\subsection{SETH-A Results}

The FMMS neutron sum for the entirety of SETH-A can be seen in Figure 7 along with the vertical profile of the main four columns.

\section{Column}

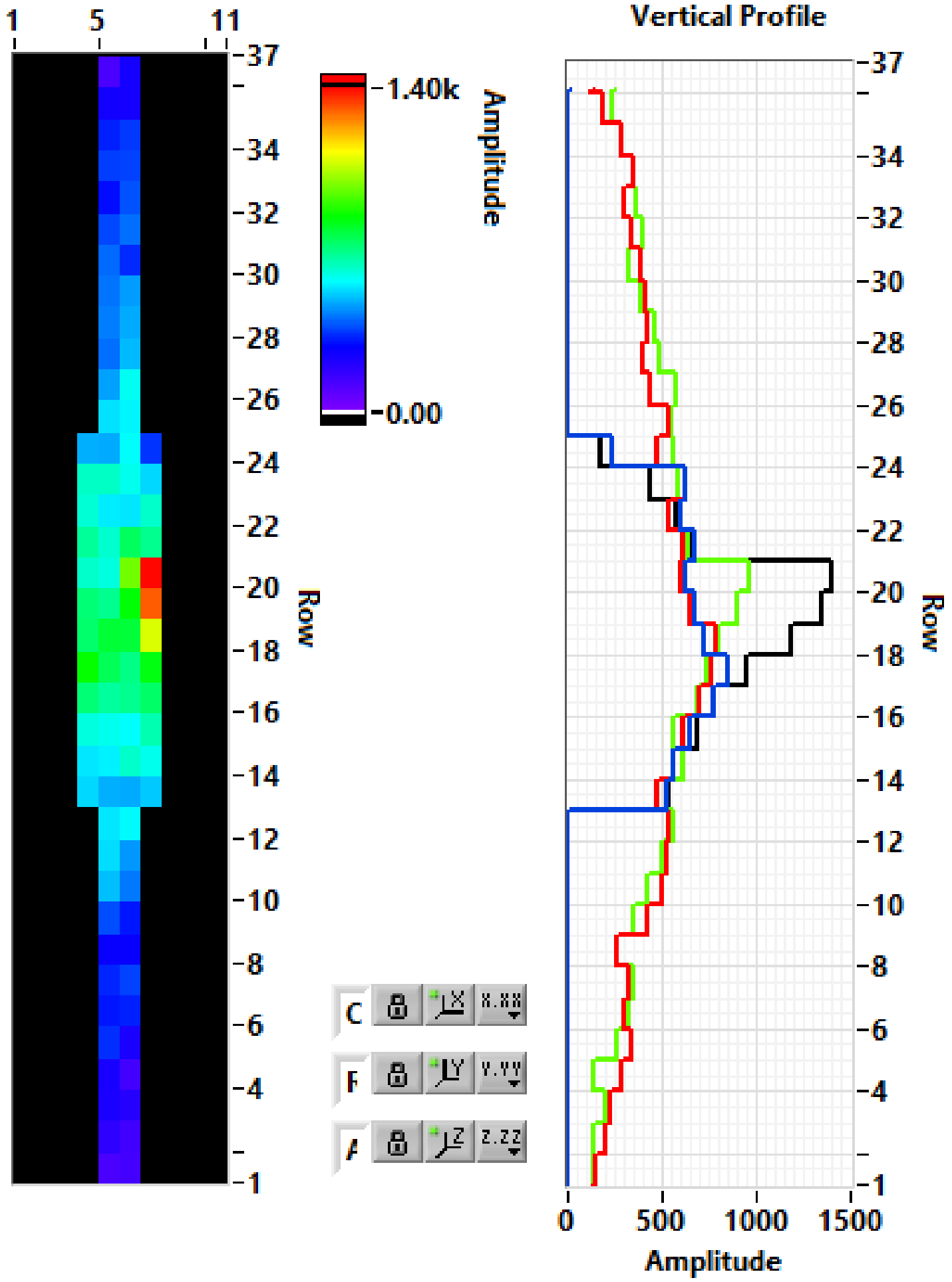

Figure 7. SETH-A FMMS neutron sum intensity plot and vertical profile for the central columns. 
The intensity plot and the vertical profile show the specimen centered on the vertical plane and off-center (to the right) on the horizontal plane of the FMMS field of view. In the vertical profile the black line represents the rightmost column followed by green, red and blue to the left. Only three detectors have an amplitude over 1000 counts, these correspond to detectors in front of the specimen. These detectors $(197,187$ and 177) are shown in Figure 8 along with the reactor power as a function of time. This figure shows the direct relationship between reactor power and detector count rate, which sharply decreases as the power is reduced by $365 \mathrm{Mw}$ in one second.

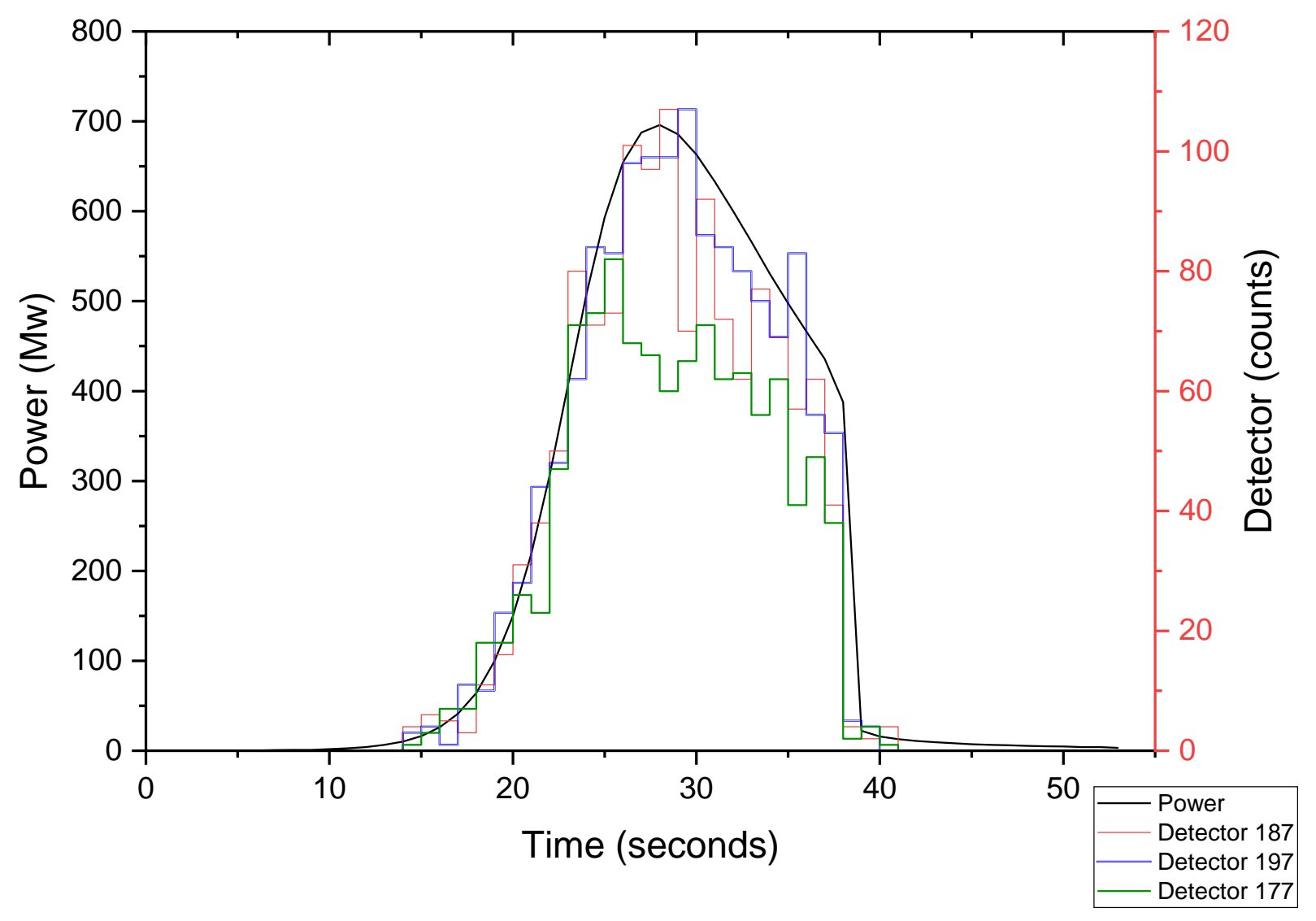

Figure 8. Profiles of selected FMMS detector count rates (counts per $1 \mathrm{~s}$ ) and reactor power over the duration of the SETH-A transient.

Snapshots of the FMMS neutron sum intensity plot at different times throughout the transient give a better understanding of how the detector array's response changes with the power profile. These snapshots are displayed in Figure 9 in different time steps. In the first 18 seconds, the FMMS had less than 50 counts per channel and many of the detectors surrounding the center of the field of view were still below 20 counts. The highest reactor power was reached at approximately 28 seconds which is reflected in the change of amplitude between figure B and C. This was the first SETH transient and these results were critical to understand the position of the specimen and the FMMS field of view, as well as the detector responses and their behavior throughout the transient. 


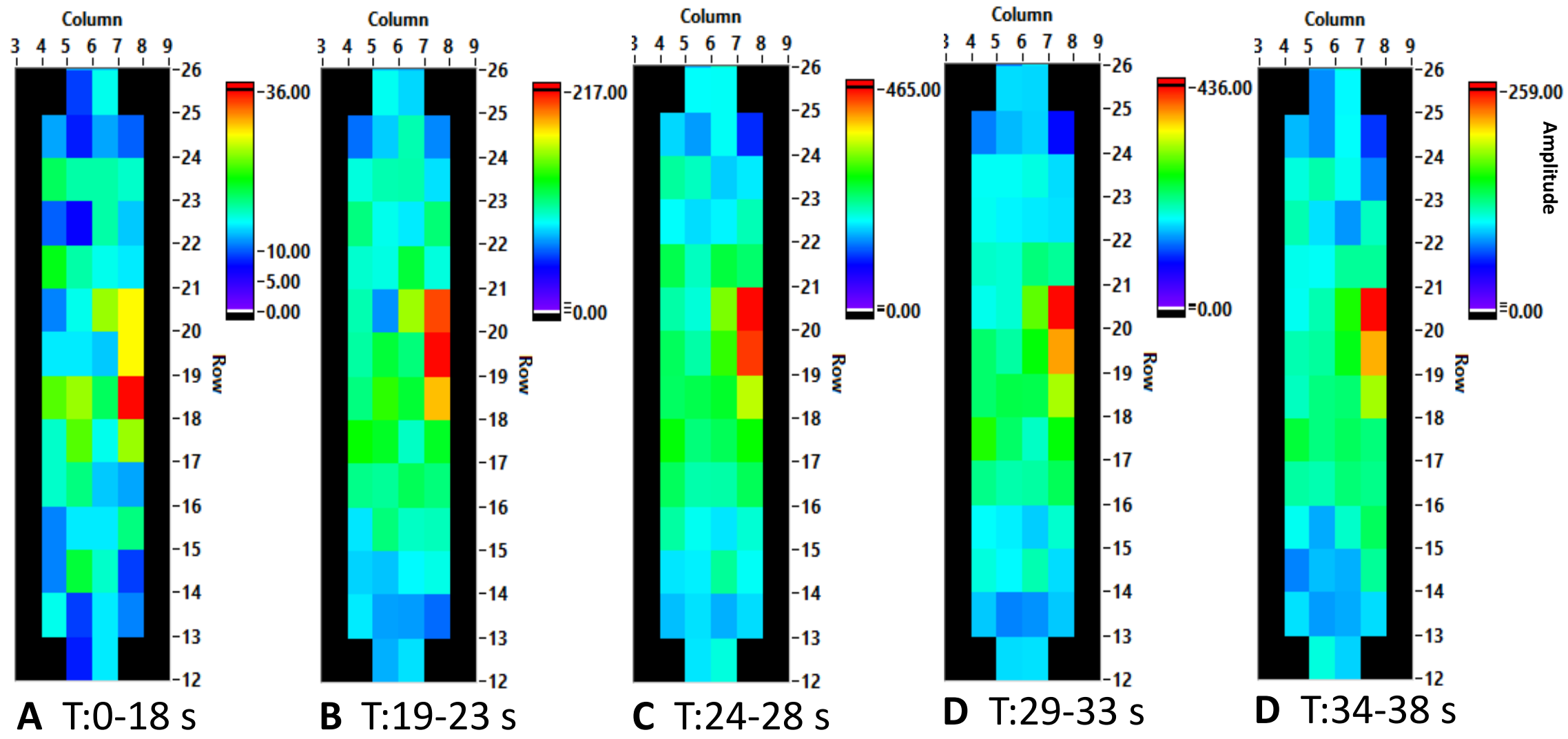

Figure 9. Snapshots of the FMMS during SETH-A at different times throughout the transient. 


\subsection{SETH-B Results}

The FMMS neutron sum for the entirety of SETH-B and the vertical profile of the main four columns is shown in Figure 10.

\section{Column}
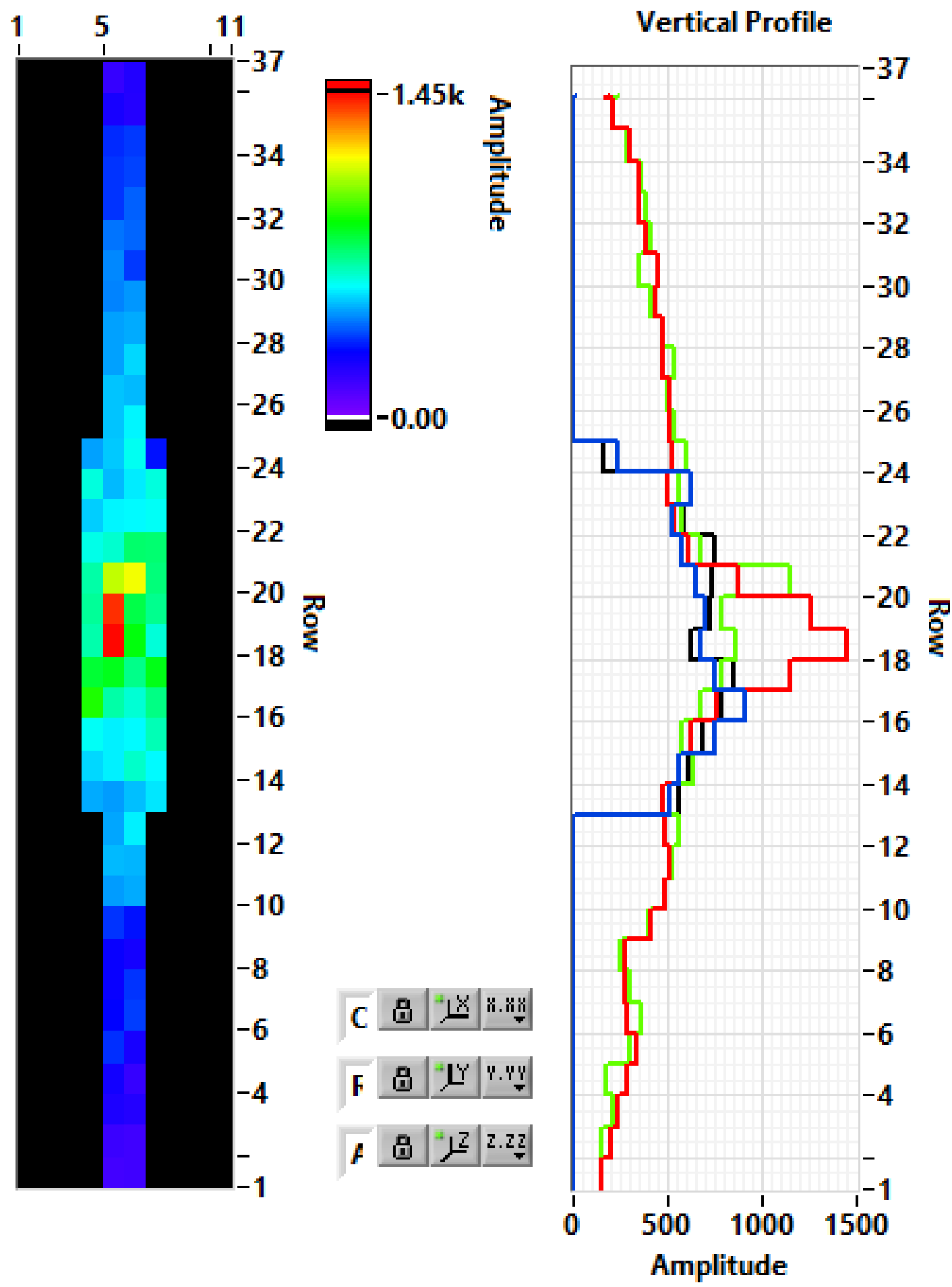

Figure 10. SETH-B FMMS neutron sum intensity plot and vertical profile for the main columns. 
Before starting SETH-B, the FMMS platform was adjusted in order to center the specimen in the horizontal plane. The transient had the same prescription as for SETH-A, leading to a similar intensity plot and vertical profile except for the higher amplitude detectors being between columns 6 and 7 and rows 18 and 21. These four detectors $(175,185,195,196)$ are highlighted in Figure 11 as a function of time along with the reactor power which has the exact shape as in SETH-A. It is important to mention the detector in column 6 (number 196) which has a high count rate and indicates that the FMMS platform was not axially aligned with the reactor, causing the specimen to appear slanted in the field of view. This same slanted effect was most likely present in the previous transient but could not be diagnosed as the rodlet was at the right-most field of view of the FMMS.

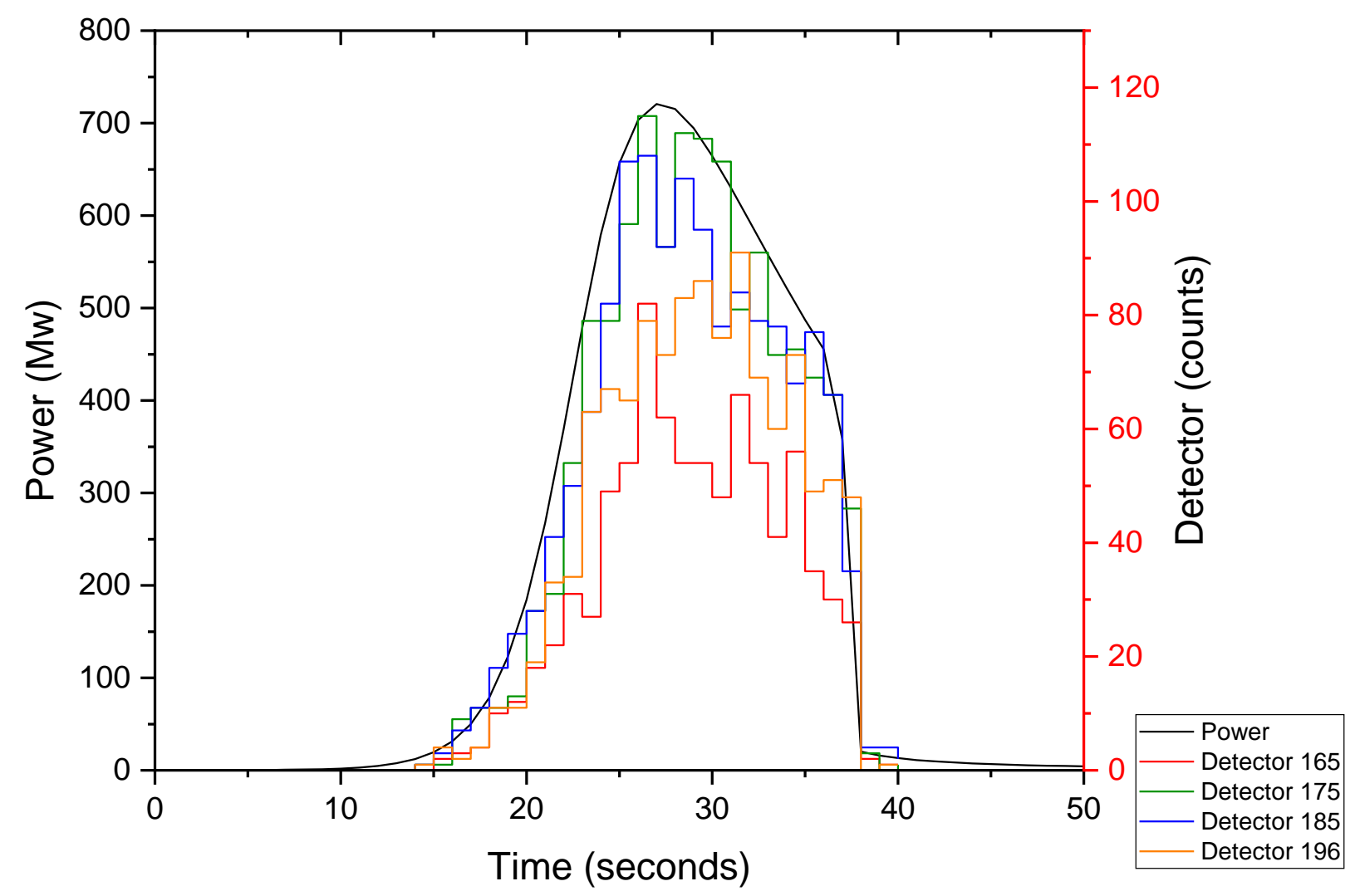

Figure 11. Profiles of selected FMMS detectors and reactor power over the duration of the SETH-B transient.

Snapshots of the FMMS neutron sum intensity graphical interface can be seen in Figure 12. These have been captured at different times than SETH-A, leading to different amplitude values. This same transient was repeated with the same $0.6 \% \Delta \mathrm{k} / \mathrm{k}$ reactivity addition to investigate different pyrometry data acquisition settings. The repetition was named SETH-B1 R2 and both its neutron sum intensity plot and central columns vertical profile are shown in Figure 13 and are very similar to those of SETH-B. This can further be seen in Figure 14, where the detector count rate and reactor power exhibit the same shape as in SETH-B. The same slanted view of the rodlet is present since the FMMS platform remained untouched during these transients. 


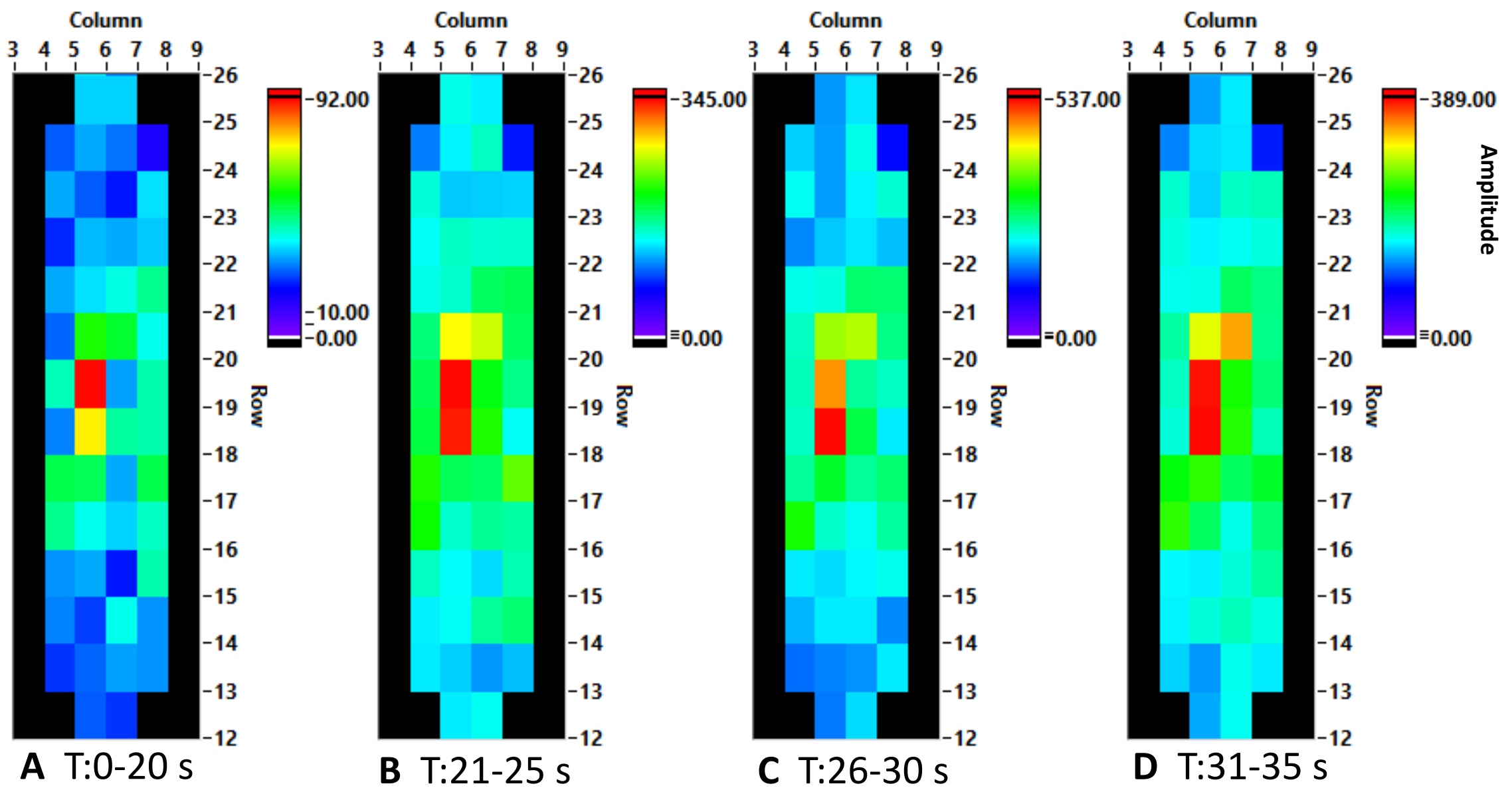

Figure 12. Snapshots of the FMMS during SETH-B at different times throughout the transient. 


\subsubsection{SETH-B1 R2 Results}

The FMMS neutron sum for the entirety of SETH-B1 R2 and the vertical profile of the main four columns is shown in Figure 13.

\section{Column}

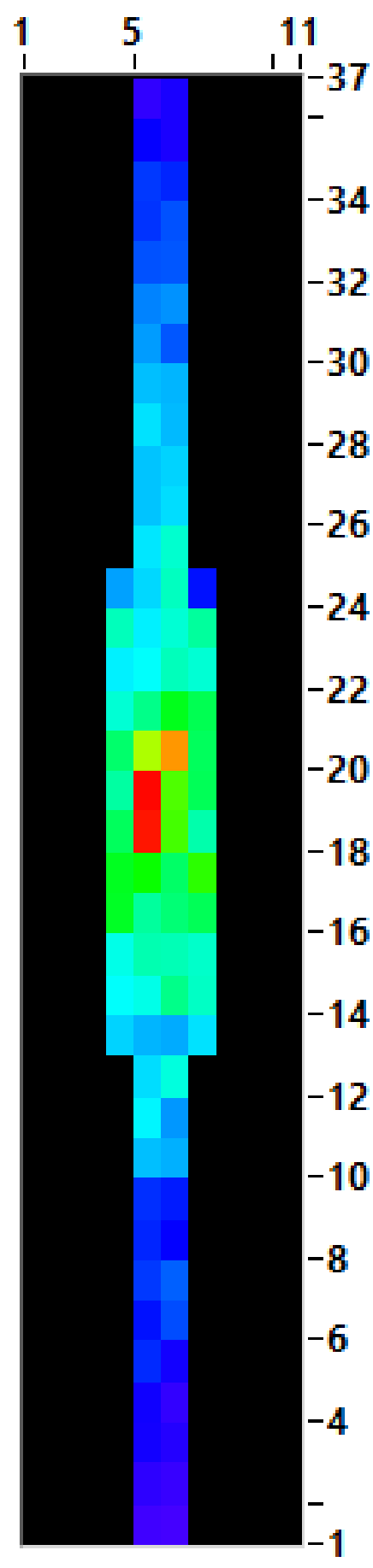

\section{Vertical Profile}

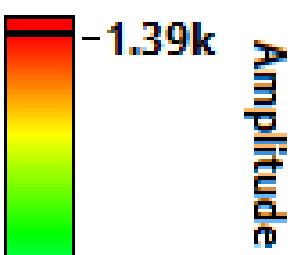

$\frac{0}{2}$

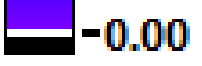

䒜

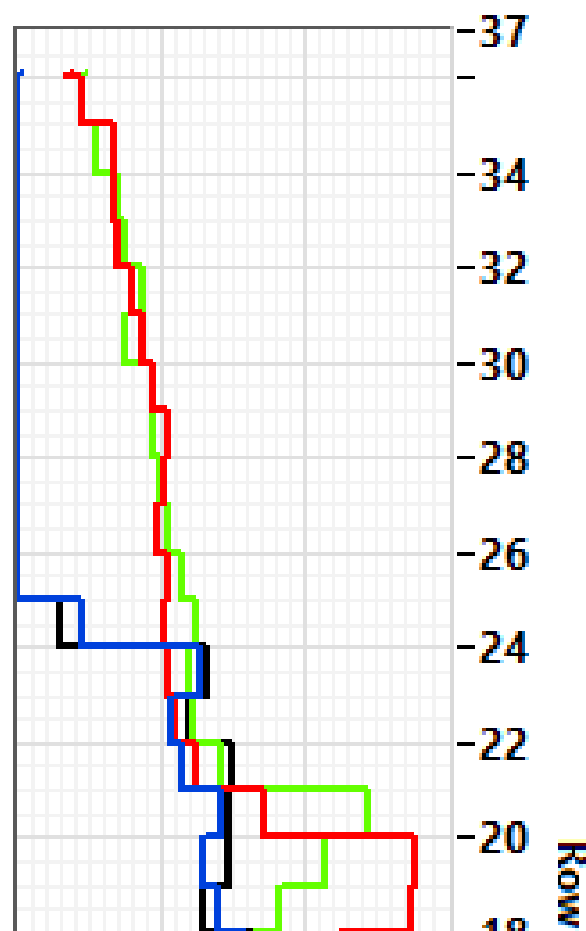

$-18$

$-16$

$-14$

$-12$

$-10$

$-8$

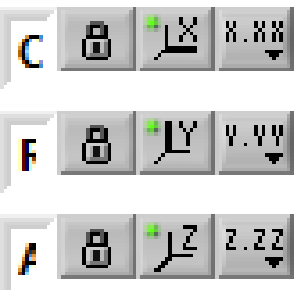

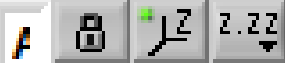

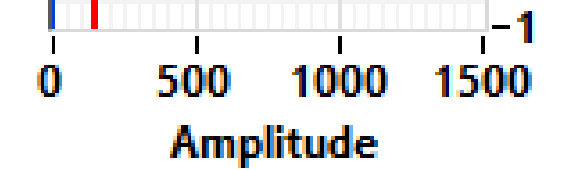

Figure 13. SETH-B1 R2 FMMS neutron sum intensity plot and central columns vertical profile. 


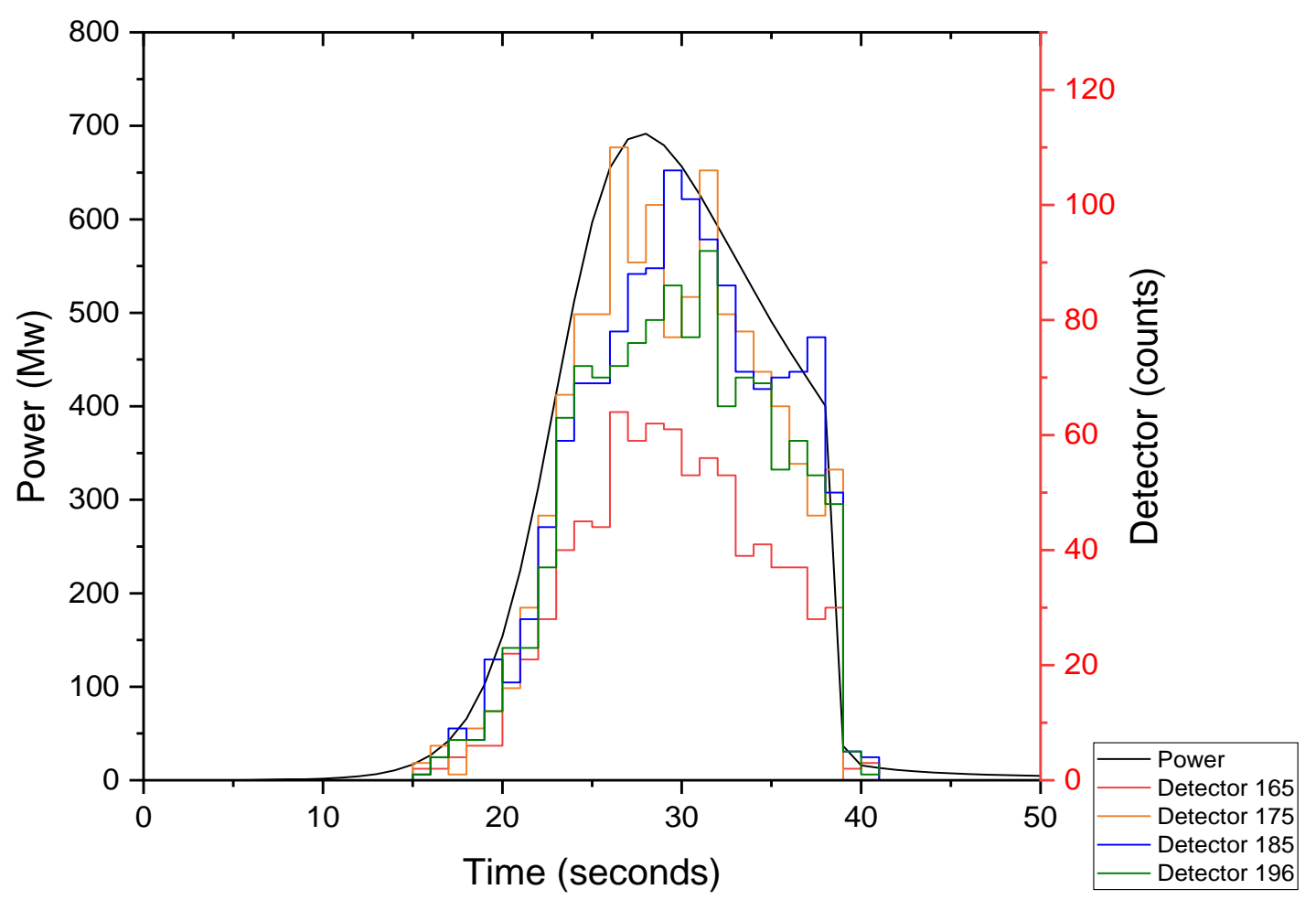

Figure 14. Profiles of selected FMMS detectors and reactor power over the duration of SETH-B1 R2.

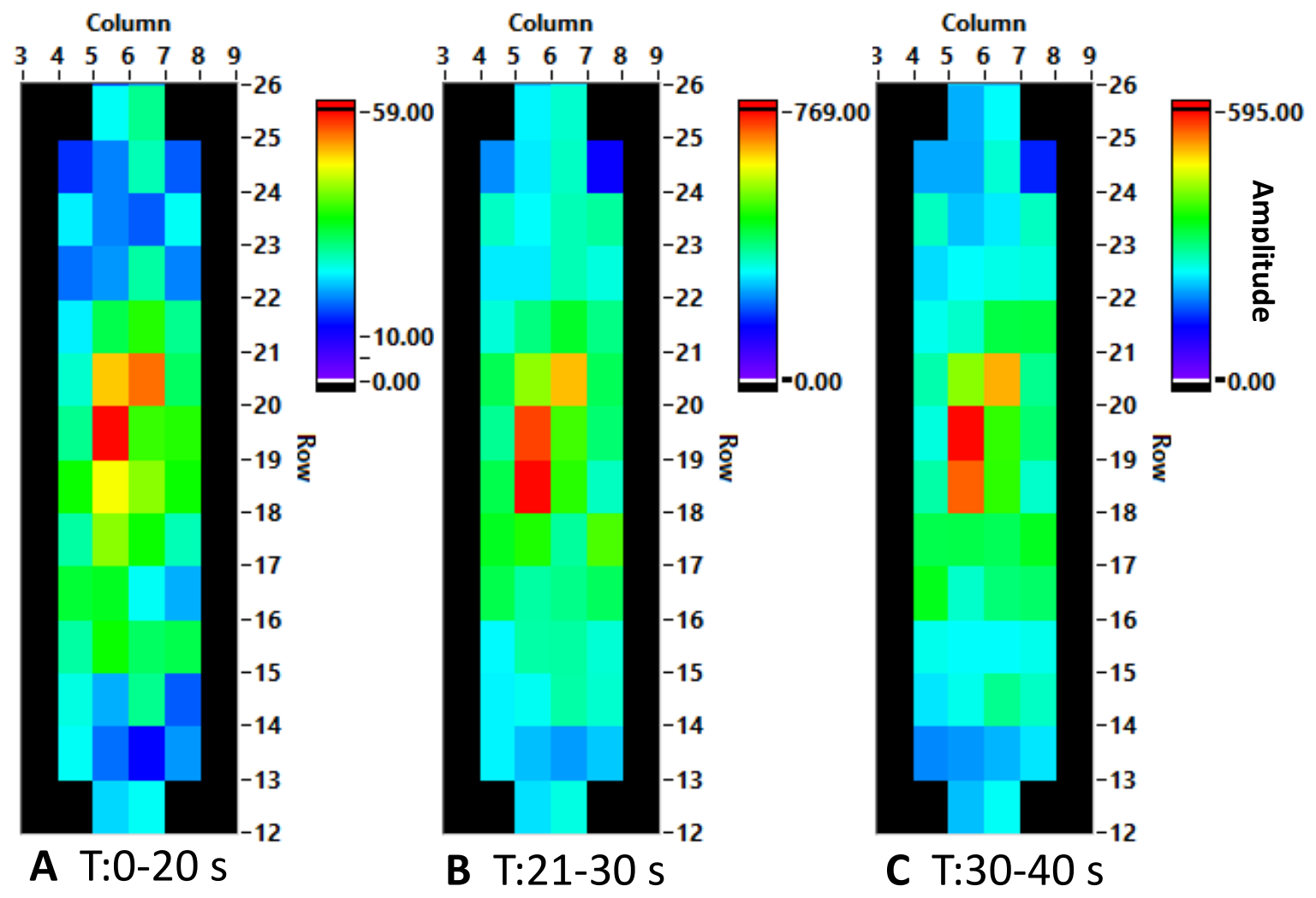

Figure 15. Snapshots of the FMMS during SETH-B1 R2 at different times. 


\subsubsection{SETH-B2 Results}

The FMMS neutron sum for the entirety of SETH-B2 and the vertical profile of the central four columns is shown in Figure 16.

\section{Column}

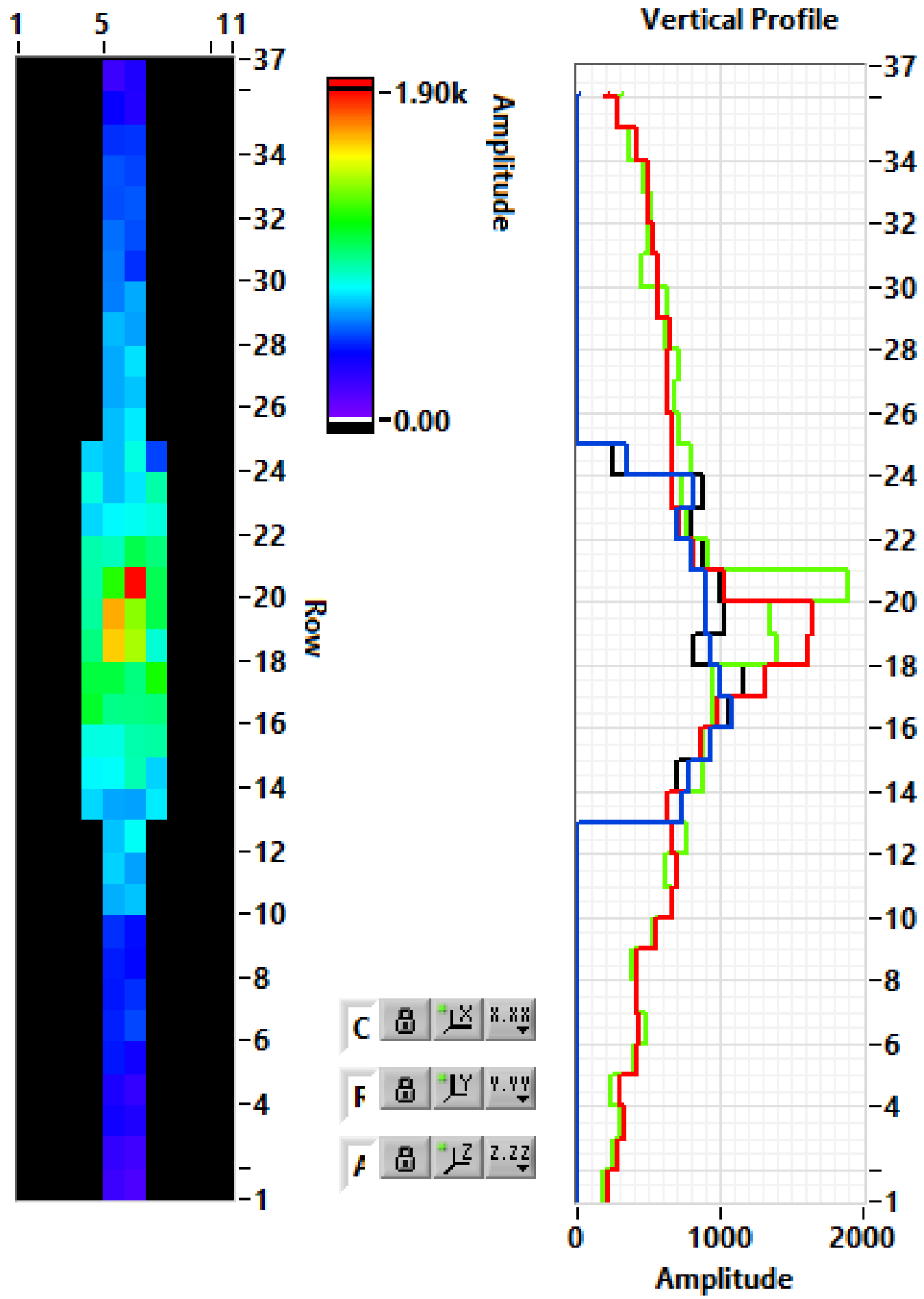

Figure 16. SETH-B2 FMMS neutron sum intensity plot and central columns vertical profile. 
The SETH-B2 transient had a higher reactivity addition, totaling $1.15 \% \Delta \mathrm{k} / \mathrm{k}$. This resulted in a higher reactor maximum power level and a different transient shape, which can be seen in Figure 17. The detectors continued to respond as expected; however, this transient was significantly shorter than the previous three with a lesser amount of data being recorded. The number of detectors selected for detailed analysis was increased to six to give an overview of the surrounding detector count rates, which are lower than the main detectors (175, 185 and 196). This can be seen in the selected profiles as well as the snapshots shown in Figure 18. The snapshots exhibit similar amplitudes as the previous transients but the time period is shorter. SETH-B2 was critical in understanding the detector response rate over a short period of time at a higher step insertion.

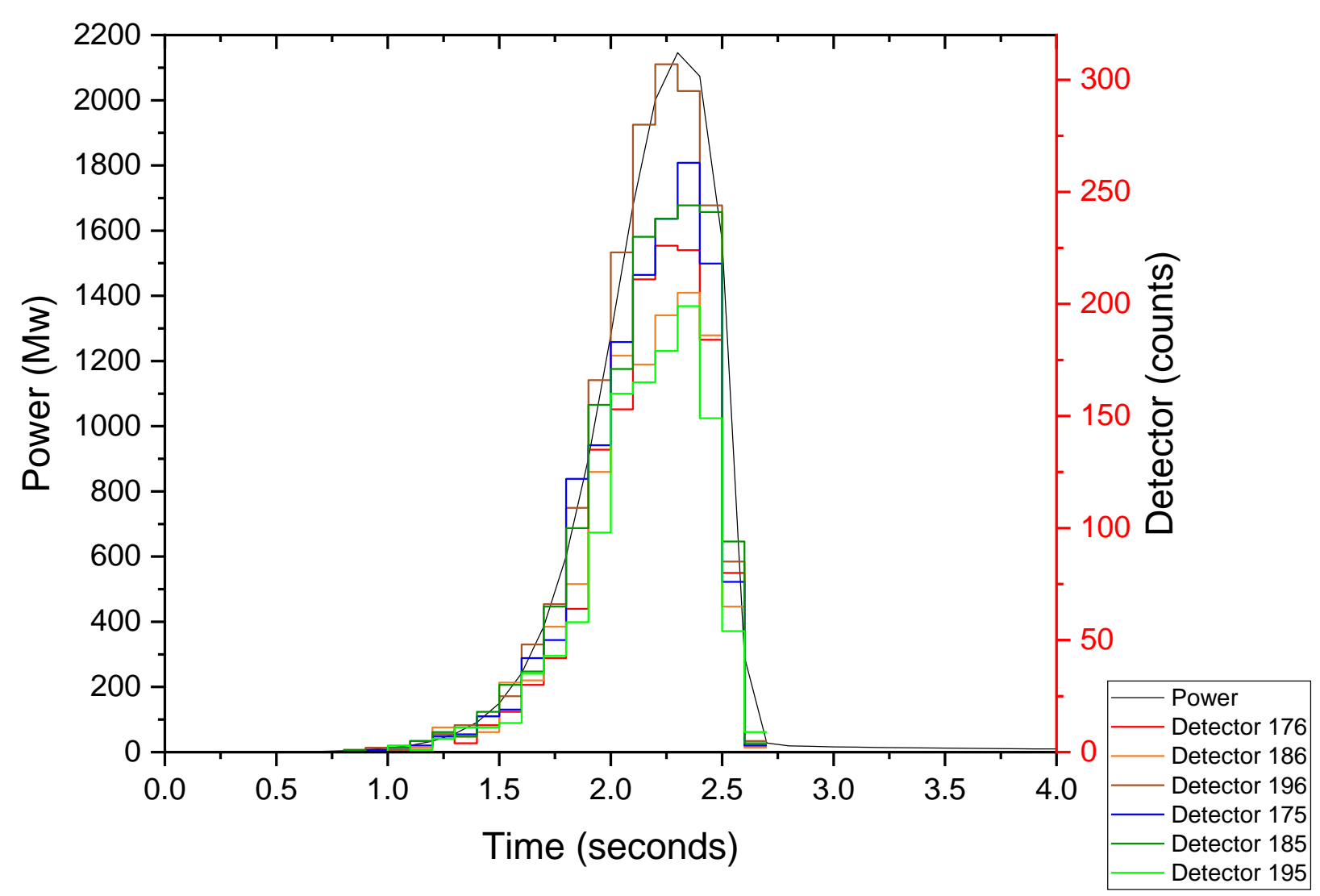

Figure 17. Profiles of selected FMMS detectors and reactor power over the duration of the SETH-B2 transient. 
Column

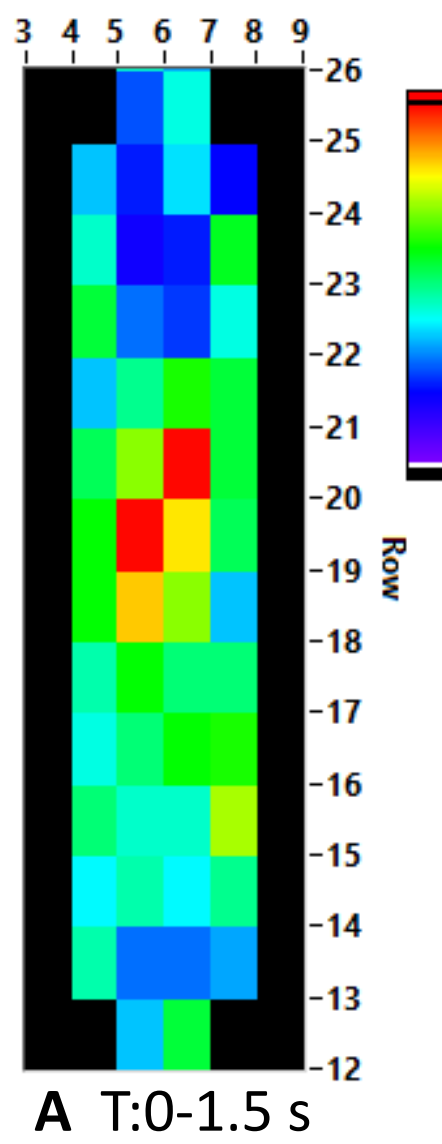

Column

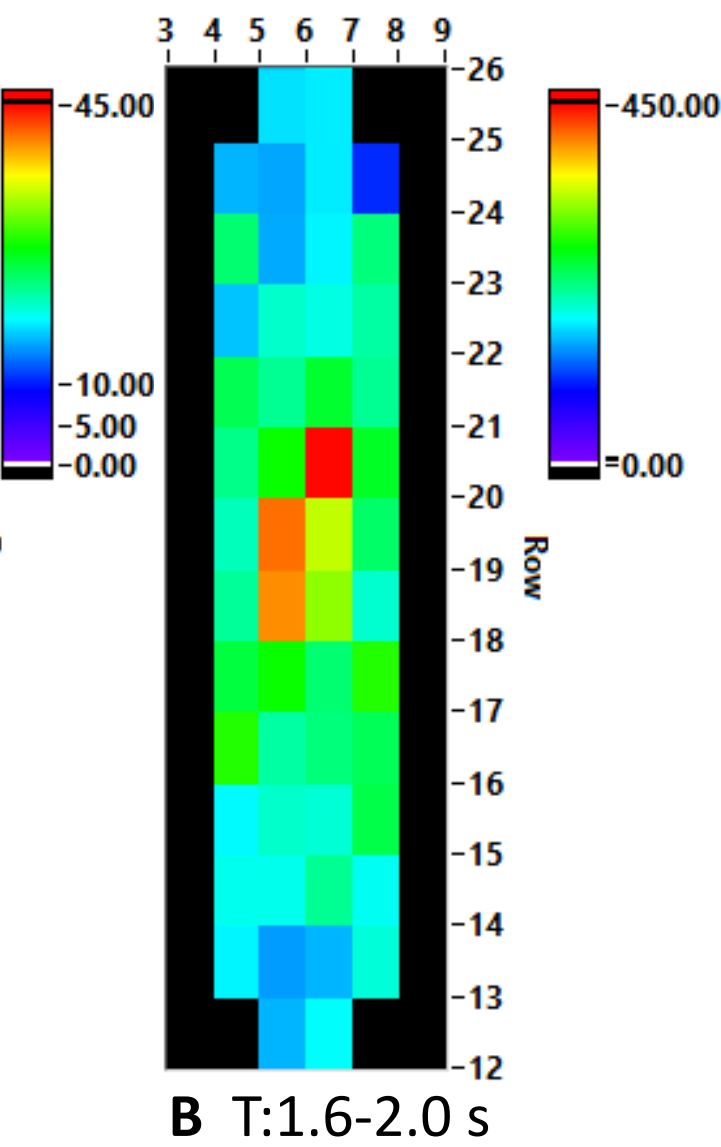

Column

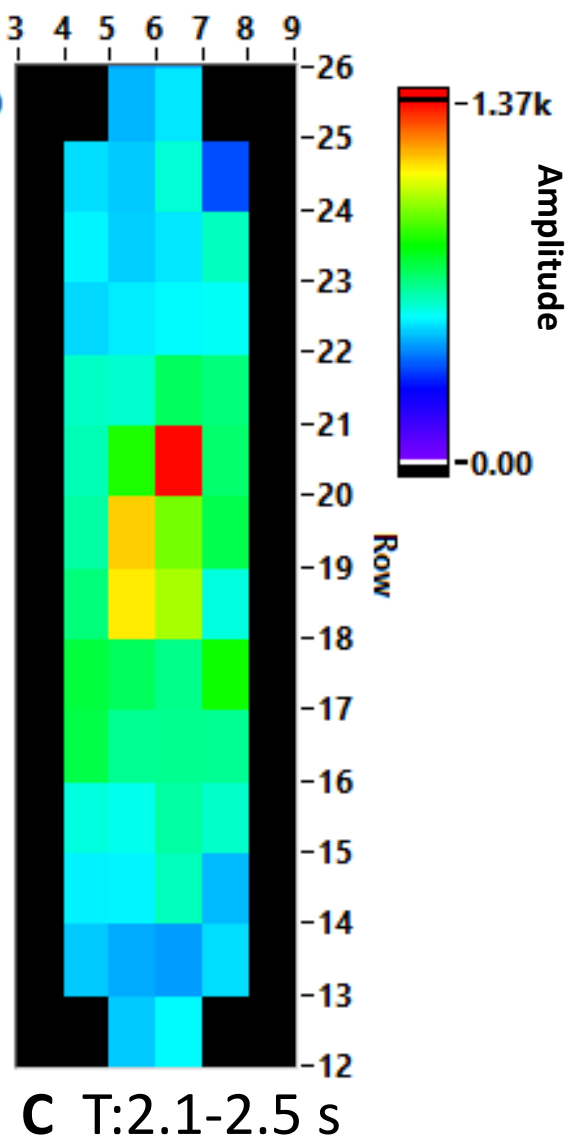

Figure 18. Snapshots of the FMMS during SETH-B2 at different times throughout the transient. 


\subsection{SETH-C Results}

The FMMS neutron sum for the entirety of SETH-C and the vertical profile of the main four columns is shown in Figure 19.

\section{Column}

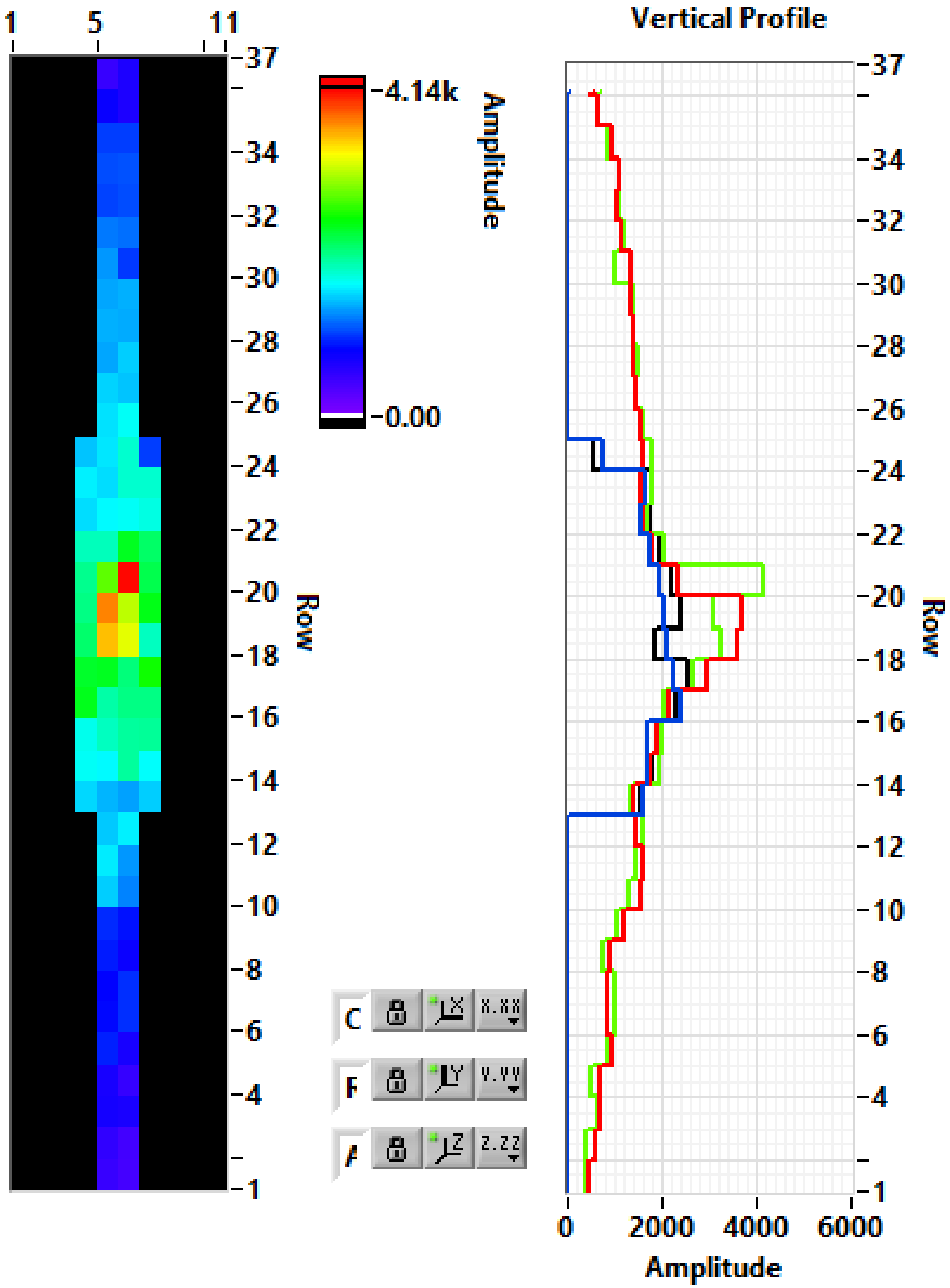

Figure 19. SETH-C FMMS neutron sum intensity plot and central columns vertical profile. 
The prescription for SETH-C was the same as SETH-B2 but it was clipped 5 seconds later as seen in Figure 20. All the previous transients were clipped shortly after the peak, showing an inmendiate drop in reactor power and detector count rate. This was not the case for SETH-C as the reactor power was decreased to approximately $200 \mathrm{MW}$ over a period of 4.5 seconds, creating a tail after the peak. During this time the six selected detectors can be seen to steadily decrease in count rate, following the shape of the reactor power.

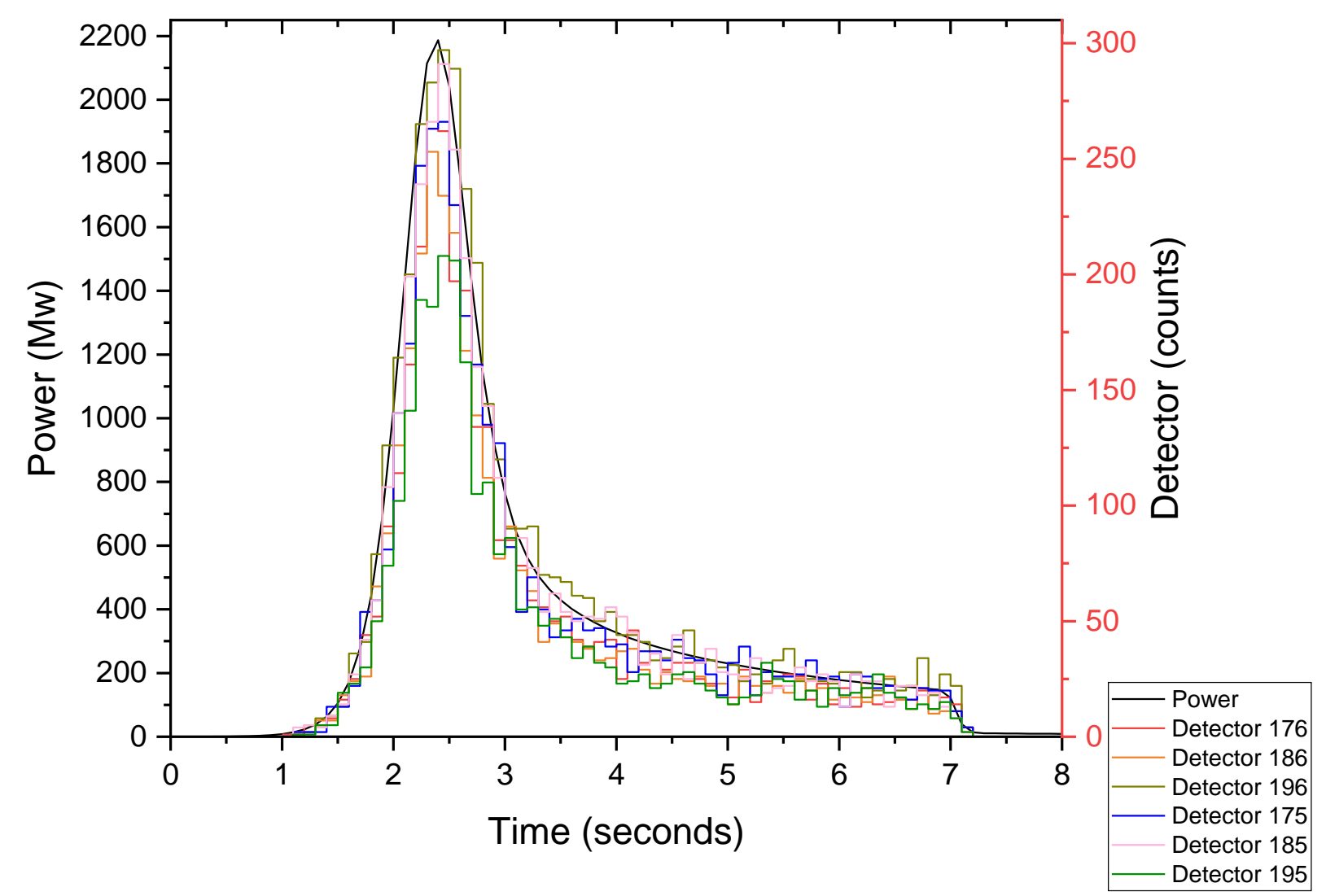

Figure 20. Profiles of selected FMMS detectors and reactor power over the duration of the SETH-C transient.

The SETH-C snapshots are shown in Figure 21, with an increased amplitude compared to SETHB2 since the snapshot times are longer. The slanted rodlet behavior is still present as no movement or changes to the FMMS were performed. The detectors with the highest number of counts remain the same throughout the transient indicating that no downward movement had been observed. 


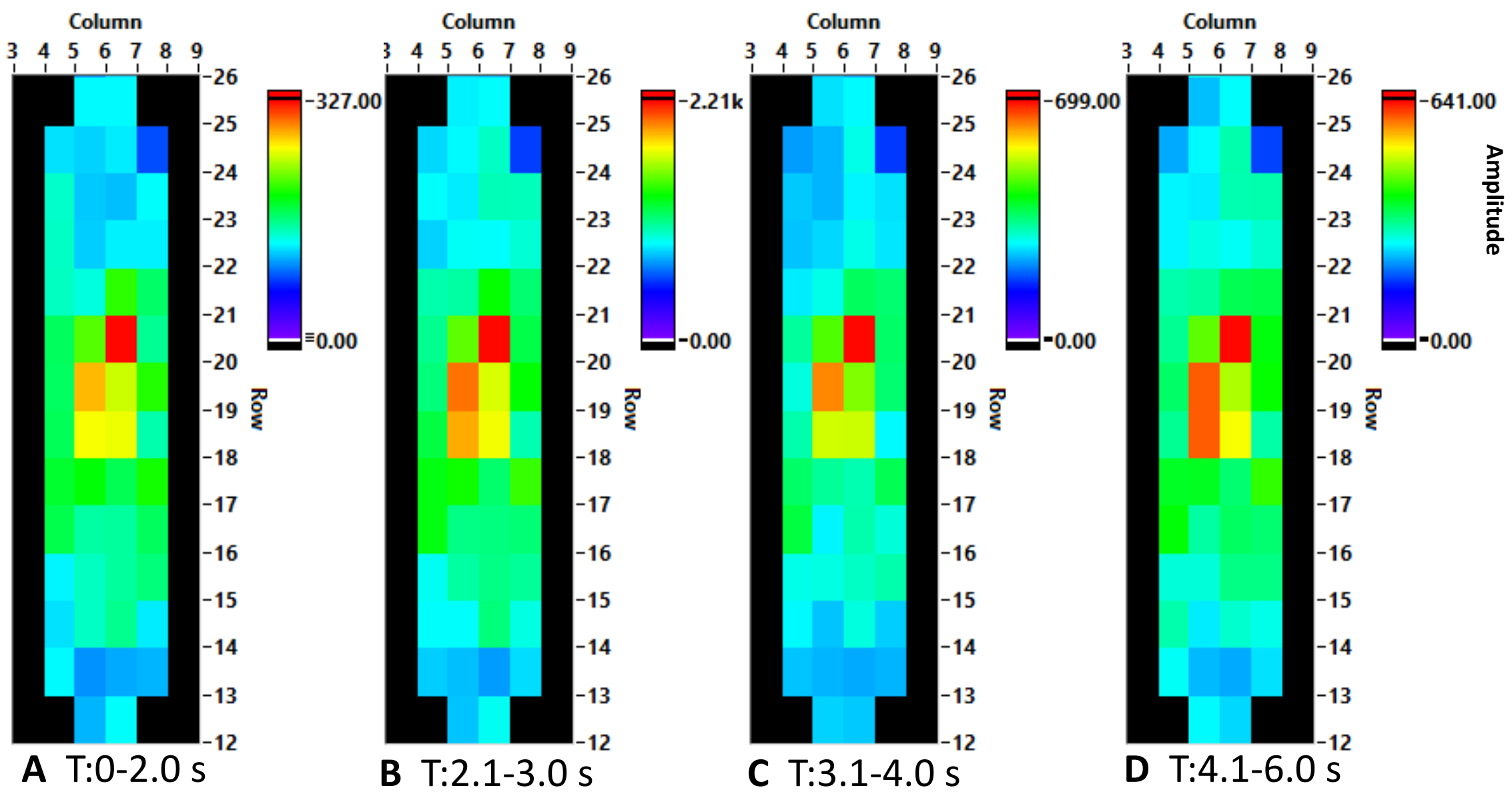

Figure 21. Snapshots of the FMMS during SETH-C at different times throughout the transient. 


\subsection{SETH-D Results}

The FMMS neutron sum for the entirety of SETH-D and the vertical profile of the main four columns is shown in Figure 22.

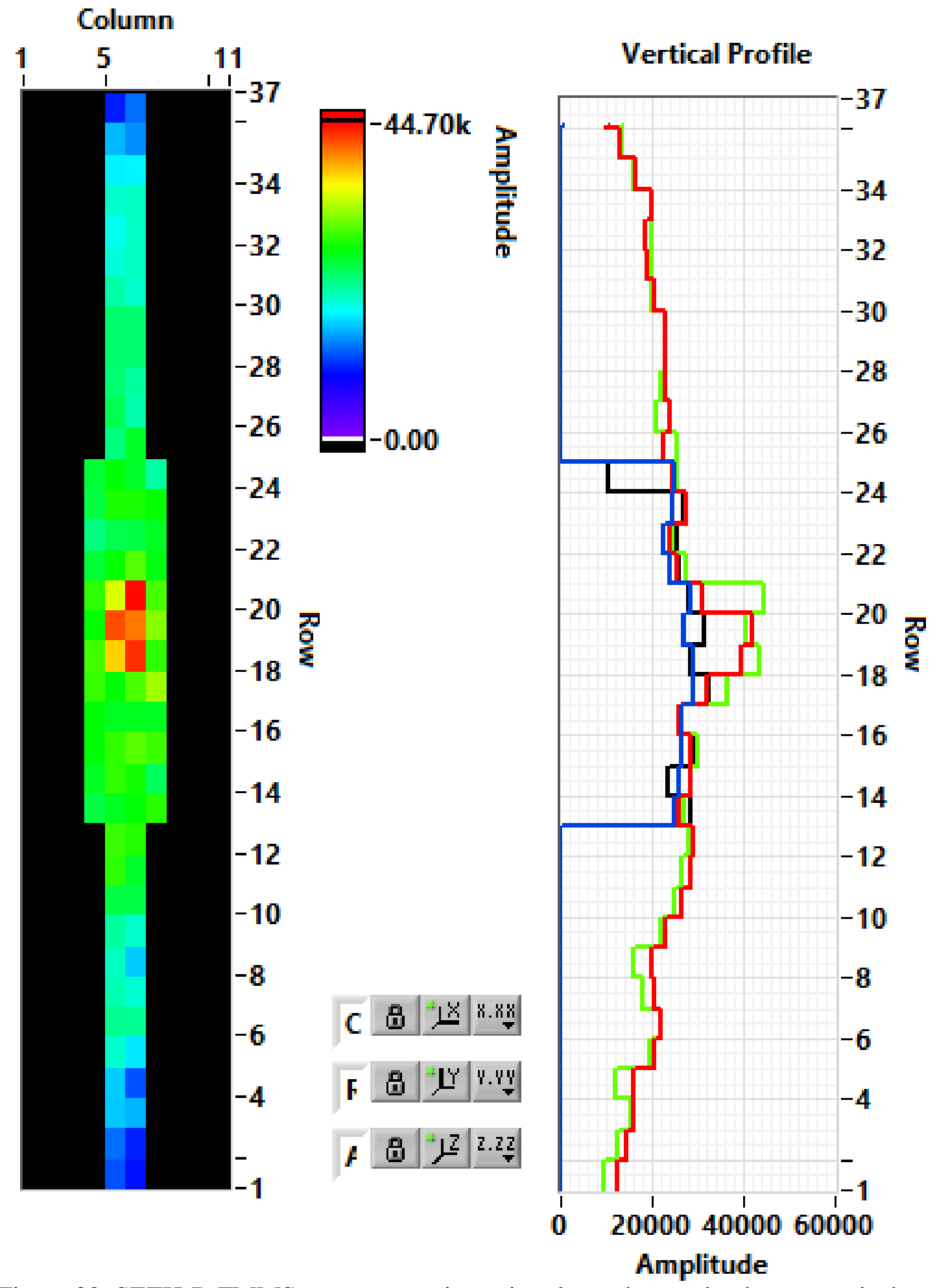

Figure 22. SETH-D FMMS neutron sum intensity plot and central columns vertical profile. 
The FMMS lead shielding was reduced from 6 inches down to 0.5 inches (11 lead sheets removed) after SETH-C. This was done to increase the detector sensitivity during the time period when zirconium cladding melting was expected, to improve the FMMS' signal and its ability to detect the expected downward motion. To achieve the cladding melting temperature the transient prescription was changed to increase the reactivity addition to $1.69 \% \Delta \mathrm{k} / \mathrm{k}$. It was expected that by decreasing the amount of lead shielding and increasing the reactivity this would saturate the FMMS detectors around peak power. This is shown in Figure 23 for the selected six detectors, each of which displays a plateau between 1.1 and 1.6 seconds when the reactor power is at its peak. In previous transients where detector saturation was not present, the detector count rate correctly tracked the shape of the reactor power. For SETH-D, upon recovery from saturation, the detectors continue to perform as expected into the tail of the transient (past 1.6 seconds). Unfortunately, the transient only lasted 3 seconds, which was not enough time for the FMMS to capture any changes in the rodlet. The snapshots shown in Figure 24 give a better understanding of what detector saturation looks like for the core view of the FMMS. Snapshot B shows significantly higher amplitudes for detectors outside of the main area of interest. Snapshot $\mathrm{C}$ shows the detectors having recovered and exhibiting a time-correlated response like the previous transients.

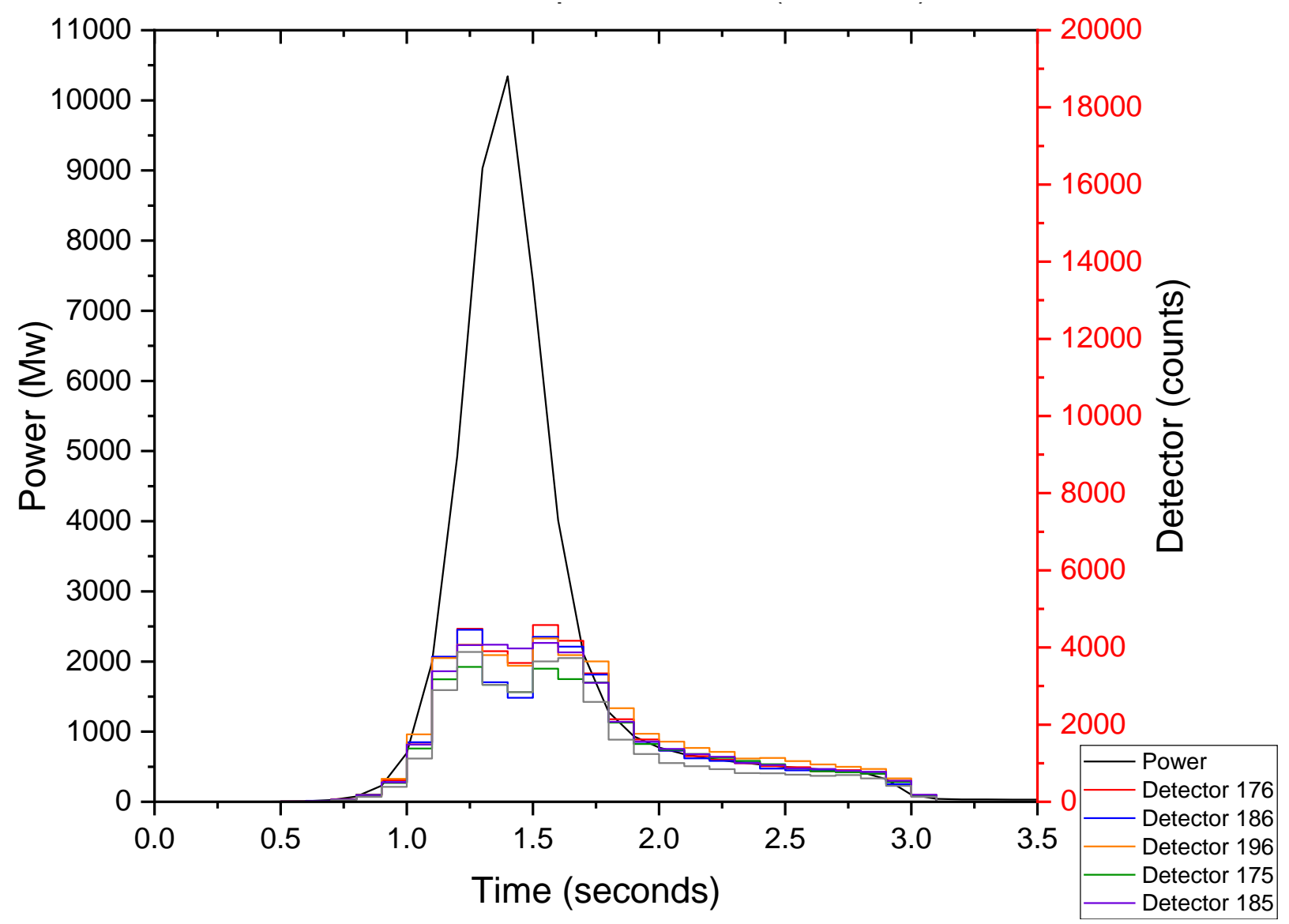

Figure 23. Profiles of selected FMMS detectors and reactor power over the duration of the SETH-D transient. 


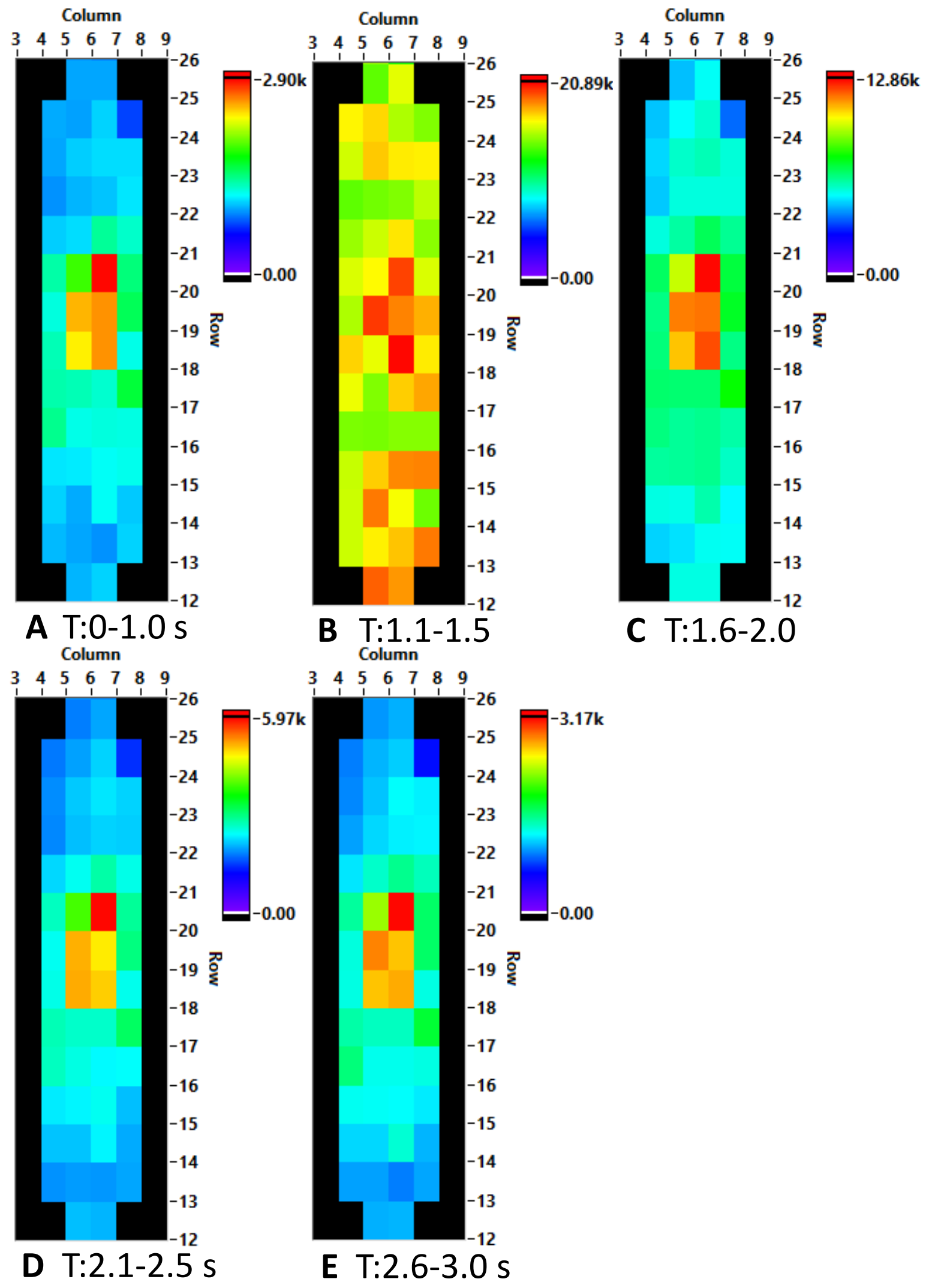

Figure 24. Snapshots of the FMMS during SETH-D at different times throughout the transient. Detector saturation was observed between 1.1 and 1.5 seconds (B). 


\subsection{SETH-E Results}

The FMMS neutron sum for the entirety of SETH-E and the vertical profile of the main four columns is shown in Figure 25.

\section{Column}
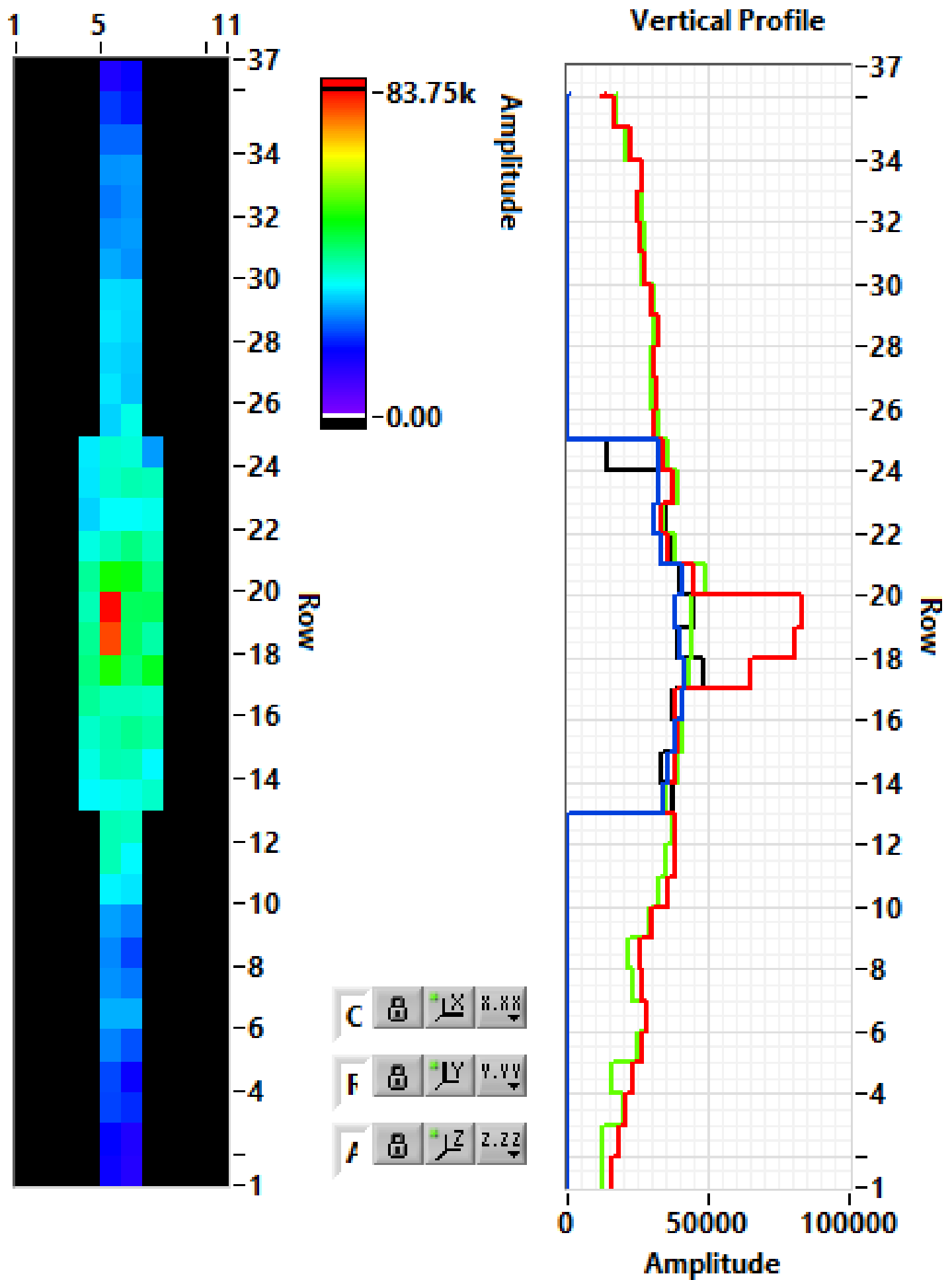

Figure 25. SETH-E FMMS neutron sum intensity plot and vertical profile for the central columns. 
SETH-E had the same reactivity addition as SETH-D but the transient time was extended to 18 seconds. The neutron sum intensity plot seen in Figure 25 presents a unique amplitude distribution as only two detectors (175 and 185) appear to have reached a high number of counts. The detector viewing the top section of the rodlet (detector 195) appears to be only above mid amplitude. To further understand this, detectors 175, 185 and 195 were selected to compare their profiles along with reactor power and the detector viewing the area below the rodlet (detector 165). The first 10 seconds of the transient are shown in Figure 26, where the previously observed detector saturation can be seen between 1.1 and 1.7 seconds. The most important feature is highlighted in the zoomed box where the detector profiles exhibit a count rate exchange between detector 195 (green) and detector 165 (orange).

The data recorded between 2 and 4 seconds is shown in Figure 27. This figure highlights the decrease in count rate for the detector at the highest section of the rodlet and the increase in signal for the detector that is below the bottom-most section of the rodlet. This observed downward motion occurred at approximately 2.8 seconds. It is important to note that since the same reactivity addition was used in SETH-D, this same motion could have been observed by the FMMS in the previous transient if the reactor power had continued past the initial 3 seconds.

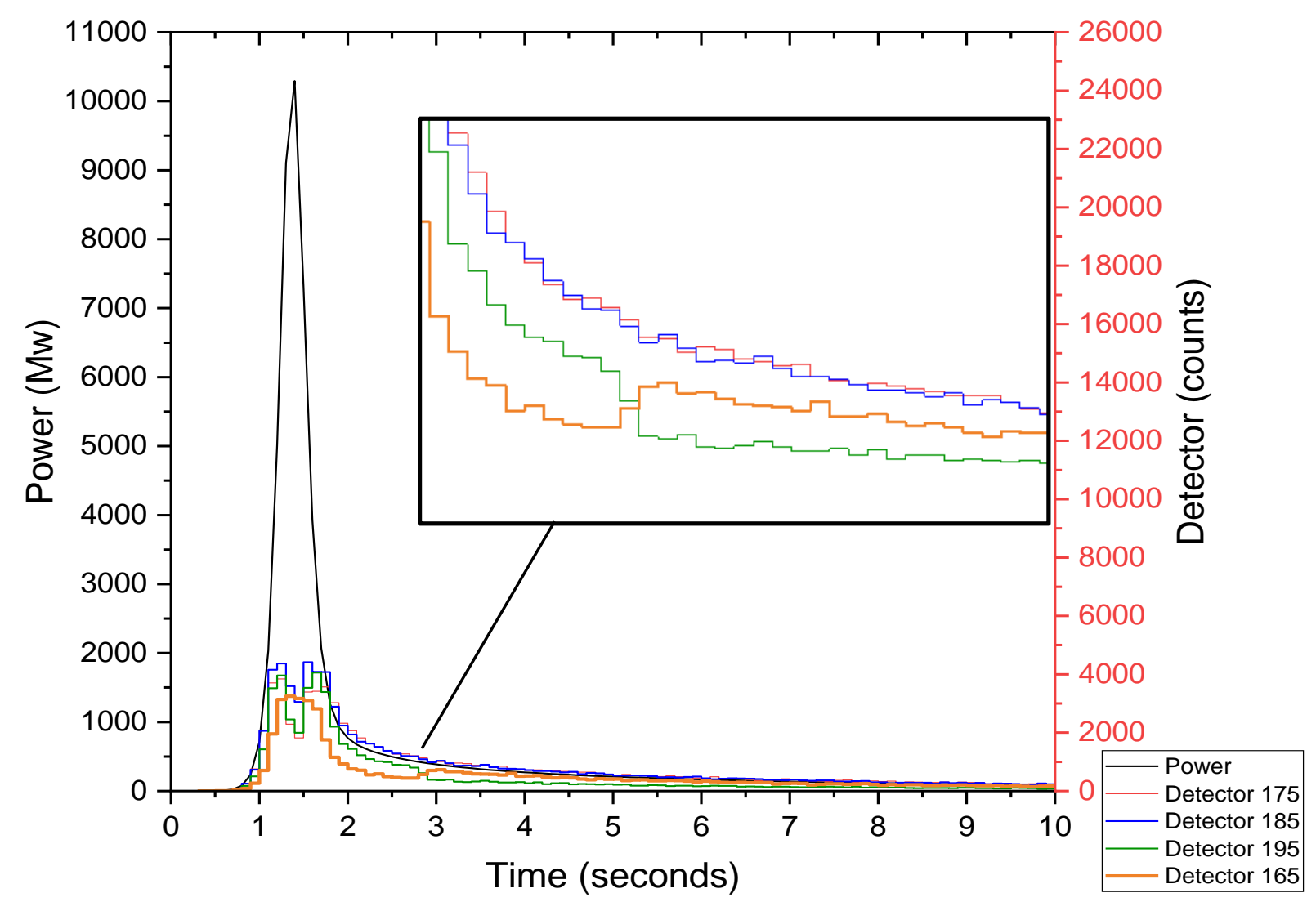

Figure 26. Profiles of selected FMMS detectors and reactor power over the duration of SETH-E. The zoomed box highlights the detector profiles at 3 seconds. 


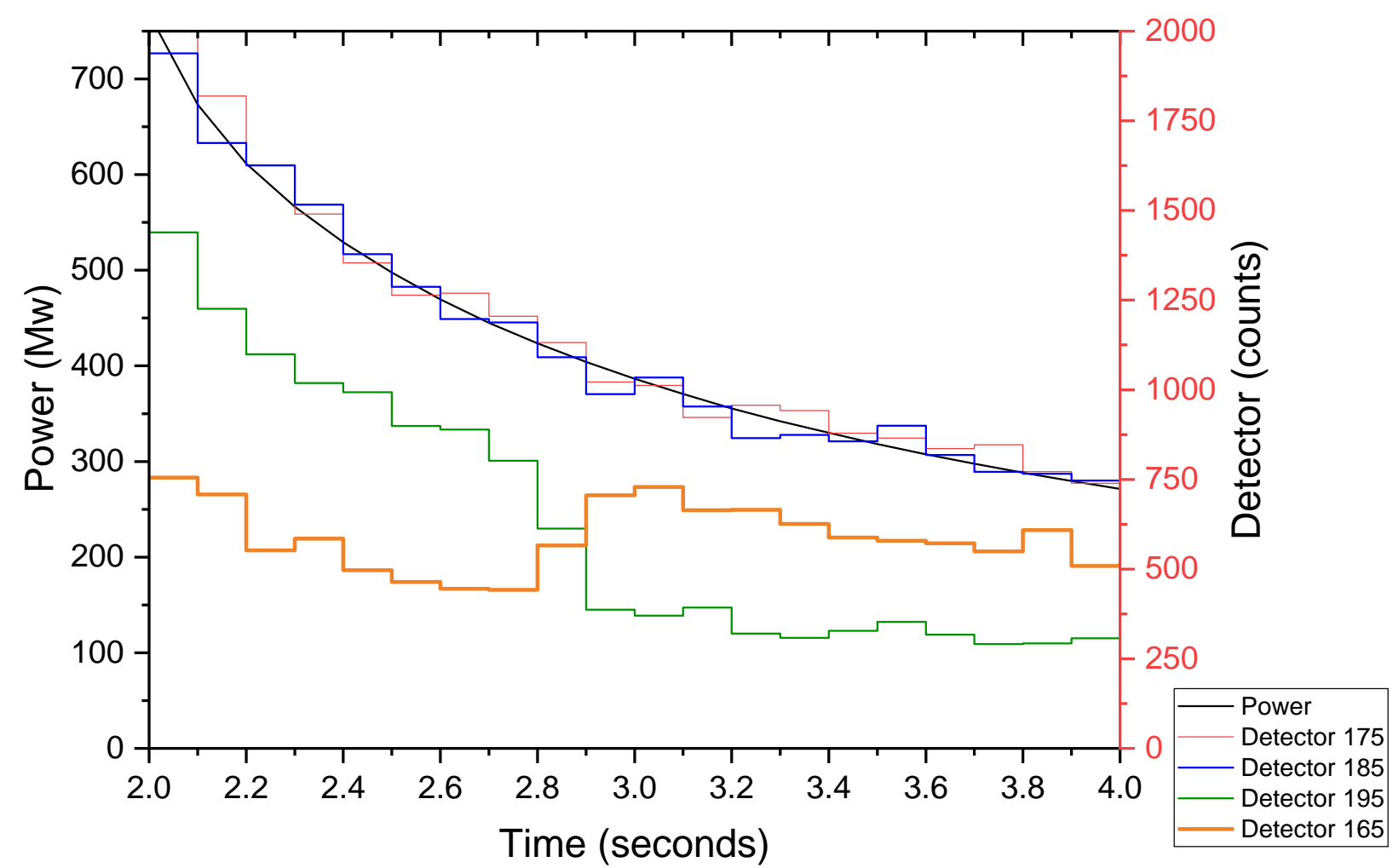

Figure 27. Enhancement of Figure 26, for time between 2 and 4 seconds, illustrating the observed downward motion of fuel in SETH-E.

The FMMS snapshots in Figure 28 provide more information focused on the first 5 seconds of the transient. The detector saturation can be seen split between Figures A \& B where three detectors in column 5 have the highest amplitude (rows 18-20). After recovering from saturation, Figure $\mathrm{C}$ shows the same three detectors with the highest amplitude. The final snapshot (Figure D) shows the change in amplitude distribution moving down one detector (down to row 17). To narrow down the time and further understand the data captured by the FMMS, snapshots around the time of expected downward movement are displayed in Figure 29. Snapshot A covers 35 milliseconds from the time previously seen in the detector profiles as the starting motion time, 2.8 seconds. The highest amplitudes are distributed in the three detectors viewing the rodlet, as expected. The next 10 milliseconds are covered by Snapshot B, which exhibits the same distribution but, given the smaller snapshot time, has lower amplitudes. Snapshot C covers the following 10 milliseconds and shows a clear change in which detectors are viewing the rodlet. Detector 165 (row 17) displays a high amplitude which was not seen in the previous snapshots and the detector viewing the top of the rodlet has an amplitude $30 \%$ less than the highest value. This helps to narrow down the time when the downward motion began. The raw FMMS data recorded in milliseconds for the two detectors of interest, plus the smoothed data after a digital filter has been applied, are shown in Figure 30. This same filtered data is shown in Figure 31 with a dashed line to highlight the time at which the count rates begin to decrease and increase for the respective detectors. This time can be assumed to be the onset of cladding melting and the start of the rodlet's downward movement. 


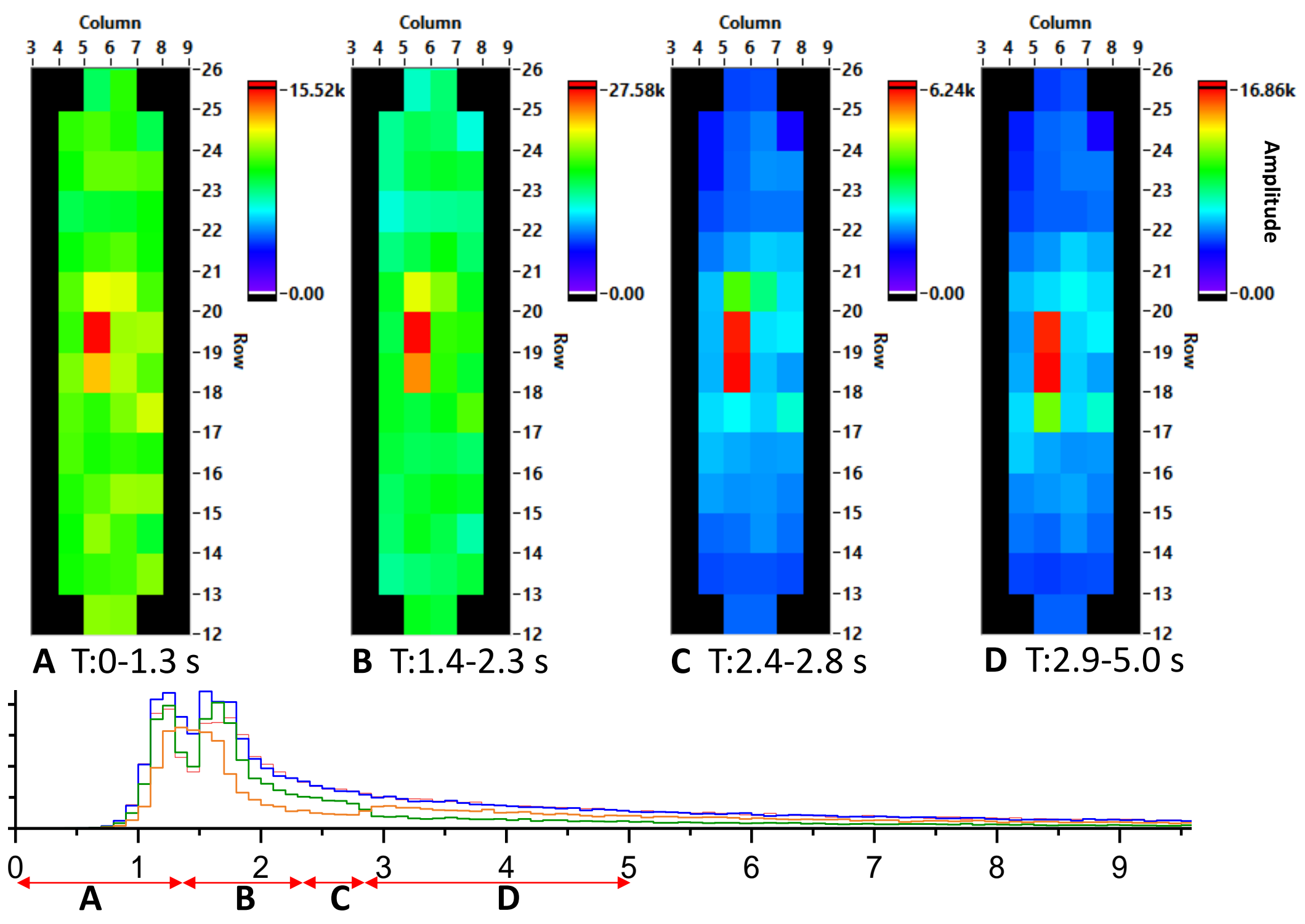

Figure 28. Snapshots of the FMMS during SETH-E at different times throughout the transient identified as A through D. The downward motion can be seen between $\mathrm{C}$ and $\mathrm{D}$. 
Column

$\begin{array}{lllllll}3 & 4 & 5 & 6 & 7 & 8 & 9\end{array}$

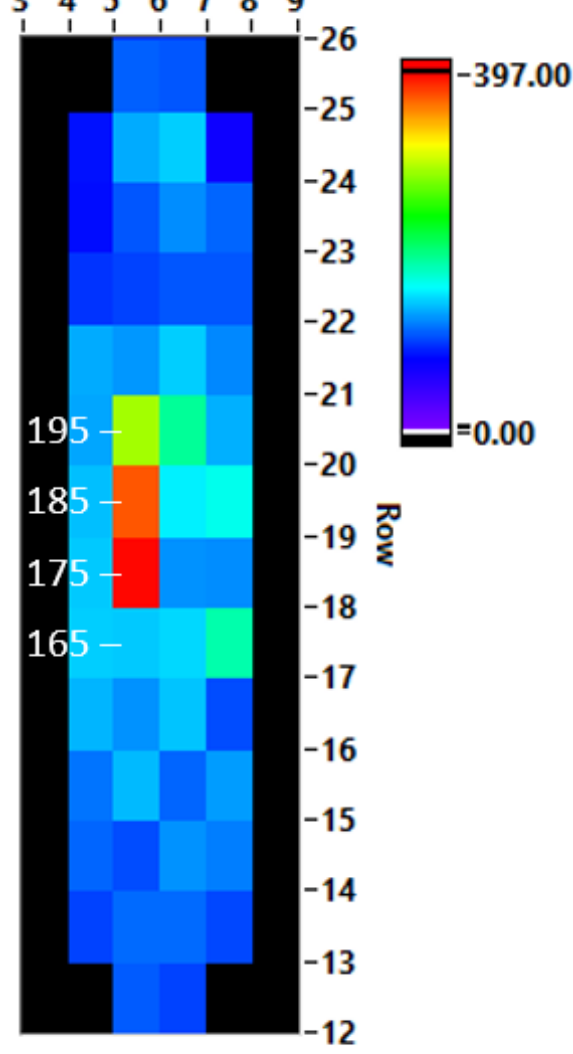

A $2.8-2.835 \mathrm{~s}$
Column

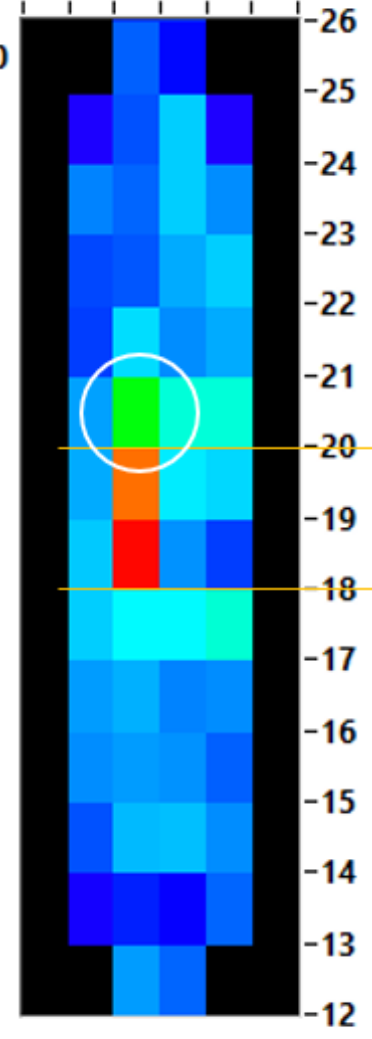

B $2.836-2.845 \mathrm{~s}$ $\begin{array}{lllllll}3 & 4 & 5 & 6 & 7 & 8 & 9\end{array}$
Column

$\begin{array}{lllllll}3 & 4 & 5 & 6 & 7 & 8 & 9\end{array}$

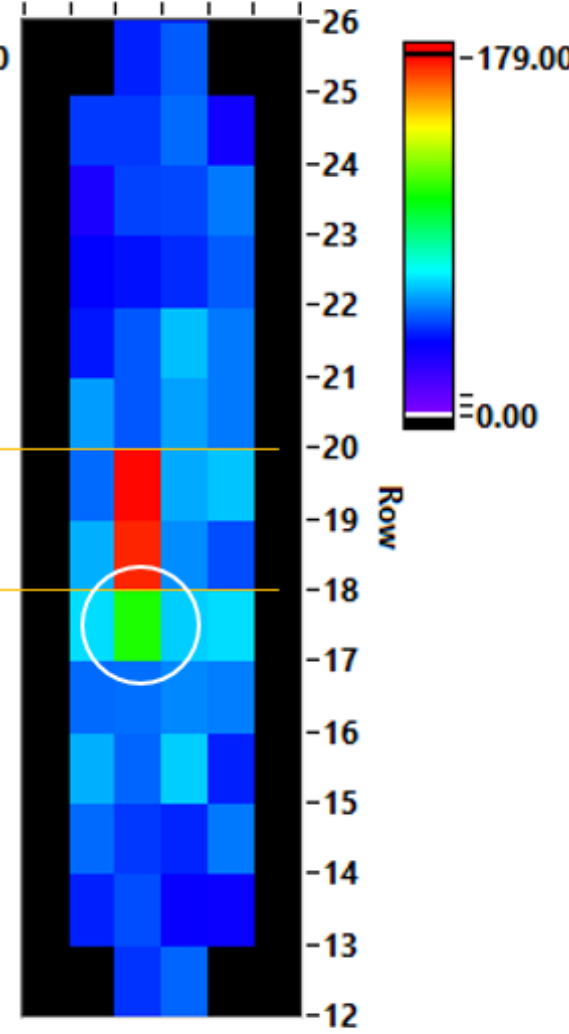

C $2.846-2.855 \mathrm{~s}$
Column $\begin{array}{lllllll}3 & 4 & 5 & 6 & 7 & 8 & 9\end{array}$

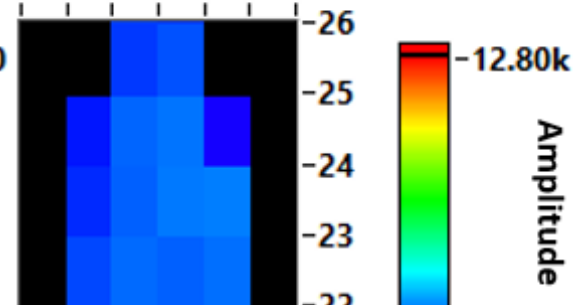

Figure 29. Snapshots of the FMMS during SETH-E at times before and after the movement of the rodlet. Detectors 165 , 175,185 and 195 are identified in Snapshot A. The downward motion is highlighted between Snapshots B and C with white circles. 


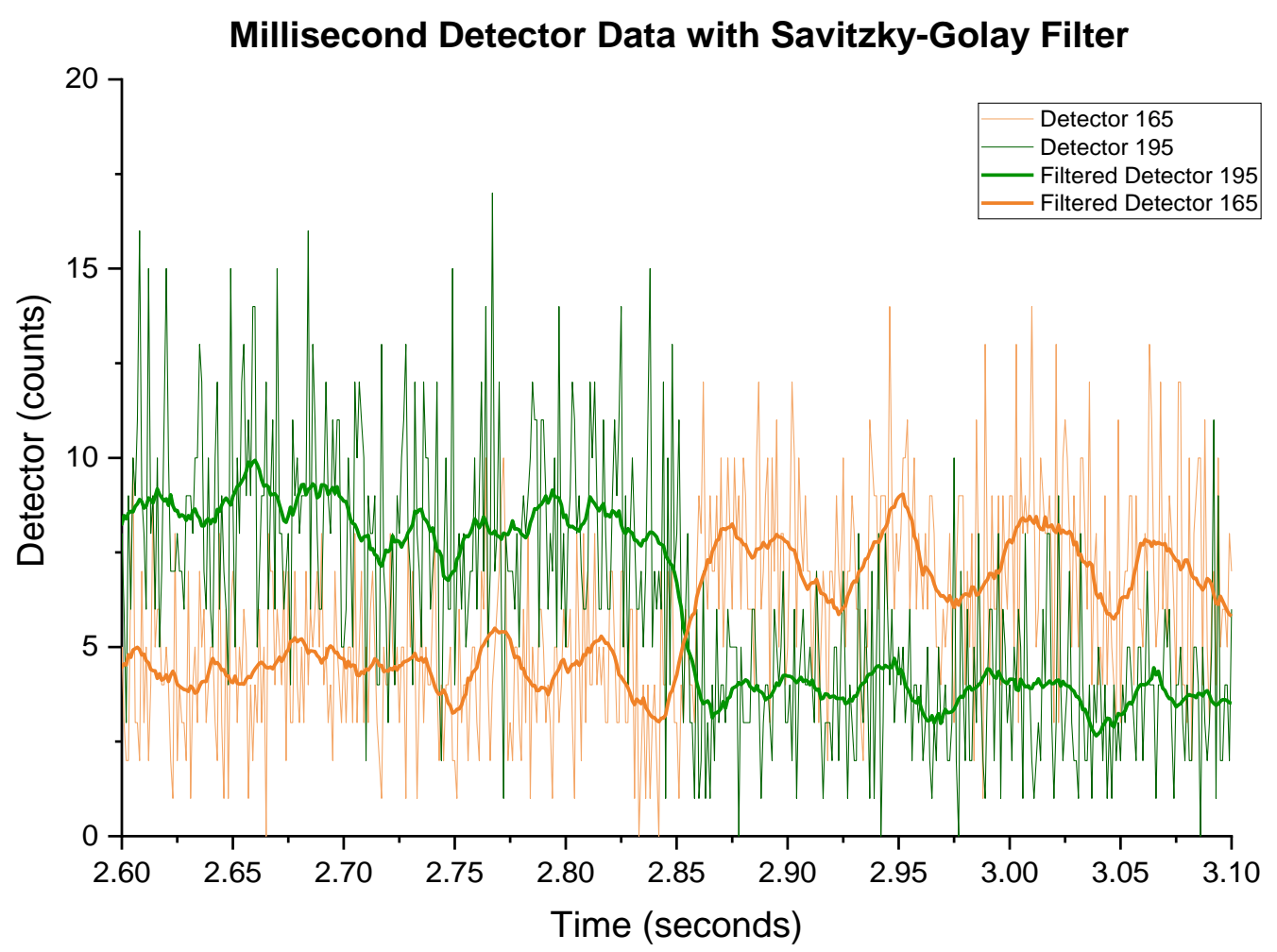

Figure 30. Millisecond data for detectors 165 and 195. The solid lines represent smoothed fits to the data using a Savitzky-Golay filter.

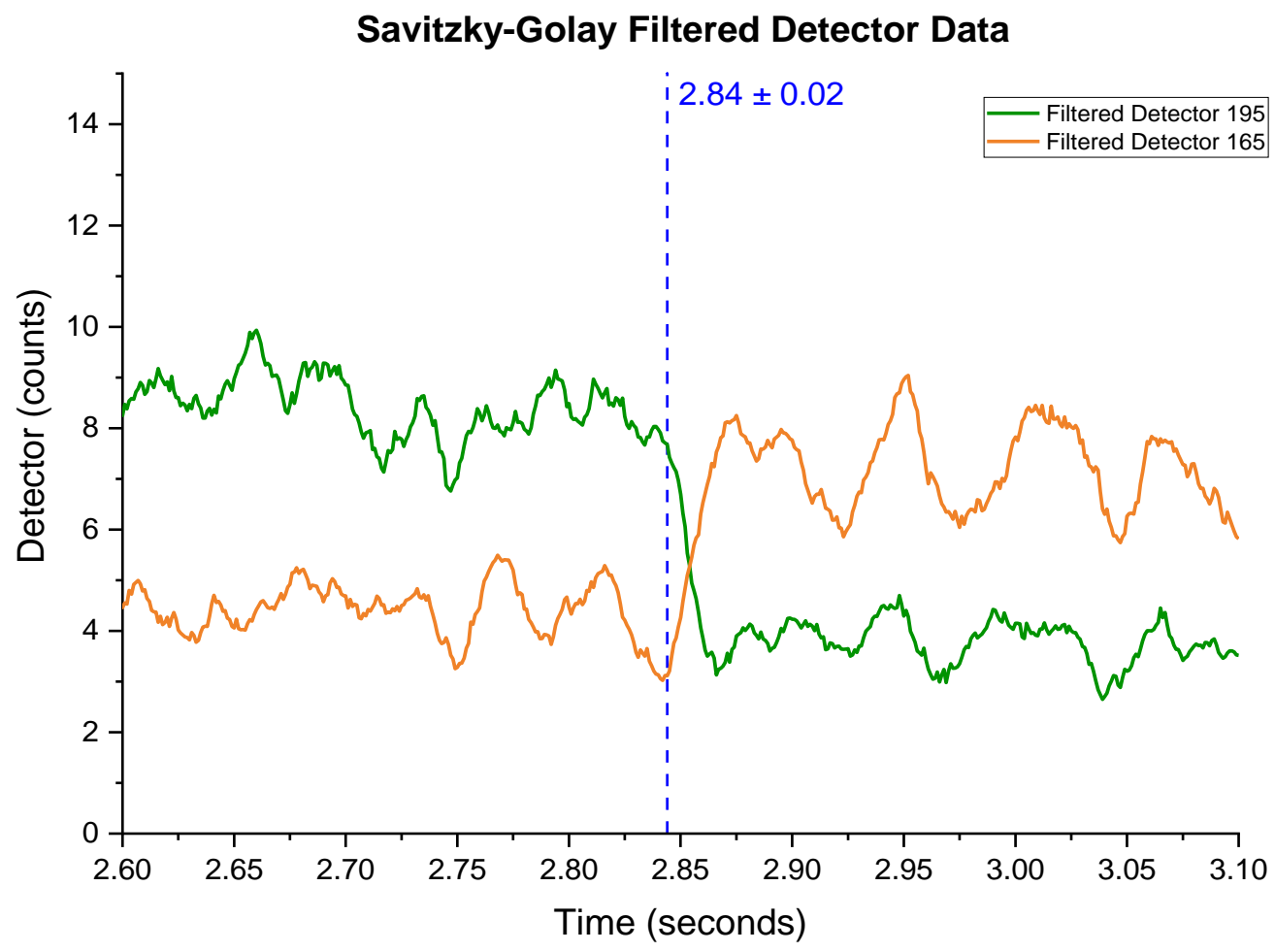

Figure 31. Filtered detector data for detectors 165 and 195. The dashed blue line identifies the approximate time when the downward motion started. 
To further comprehend the data from the FMMS and other instrumentation, post-test neutron radiography was performed on the SETH capsules. The neutron radiographs for SETH-C, -D and -E are shown in Figure 32. As previously mentioned, melting of the cladding occurred in SETH$\mathrm{D}$ but the FMMS was unable to capture the downward movement as the transient was clipped very early. The neutron radiograph of SETH-E agrees with the downward motion captured by the FMMS and shows the melted cladding.

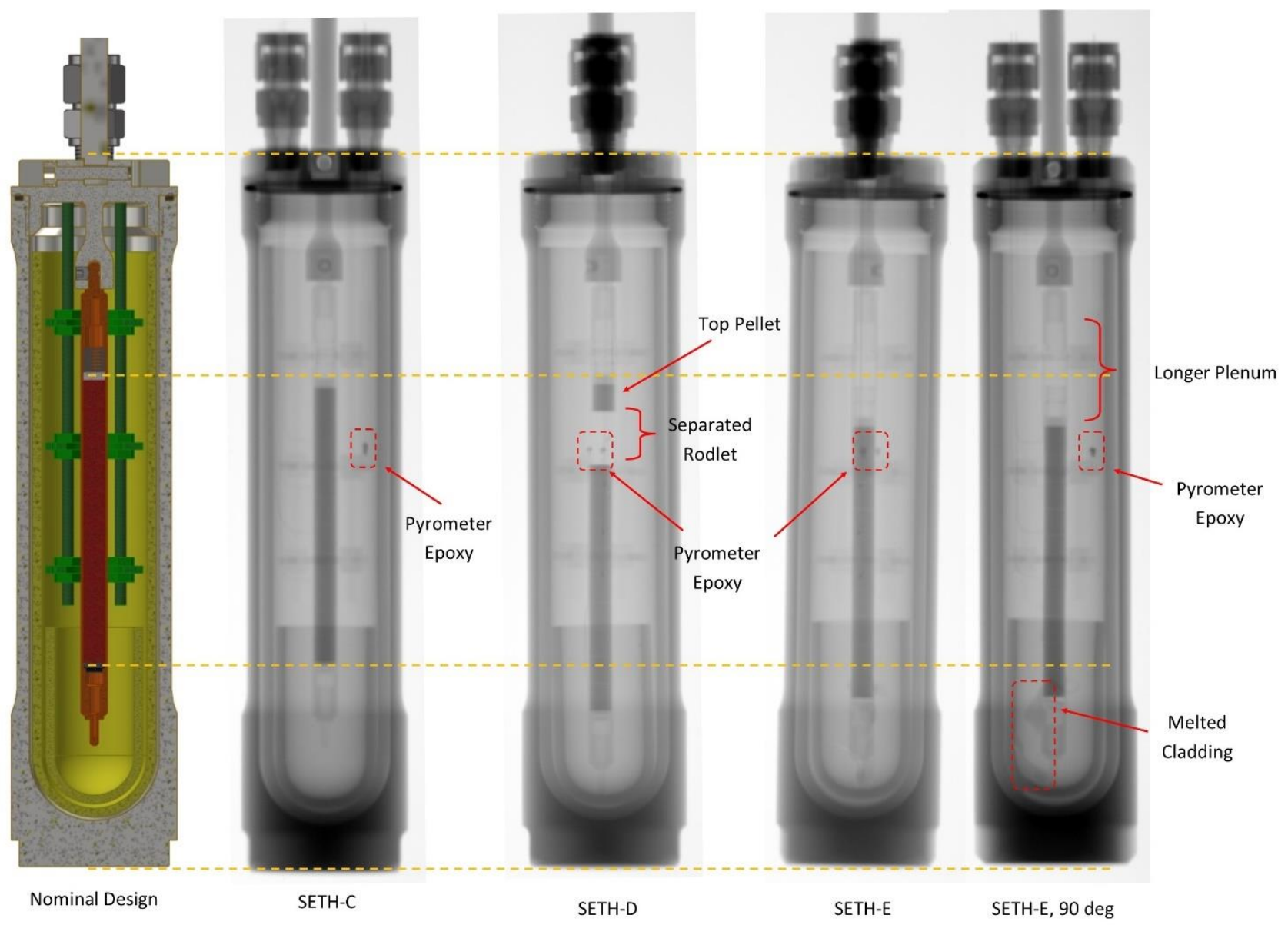

Figure 32. Post transient neutron radiographs for higher energy SETH tests [3]. 


\section{SUMMARY AND CONCLUSIONS}

The Separate Effects Test Holder (SETH) is a sub-length pressurized water reactor (PWR) fuel rod (rodlet) with zirconium alloy cladding containing ten commercial $\mathrm{UO}_{2}$ fuel pellets enriched to 4.9 wt. $\%$ in ${ }^{235}$ U. Five SETH capsules were built for a series of tests in 2018-2019, they were identified as SETH-A through -E. Prior to each transient each capsule was filled with helium gas at room temperature and roughly atmospheric pressure. Each SETH capsule hung from a large hanger rod inside the MARCH-BUSTER configuration. The rod length was chosen to allow the specimen to be axially positioned in the vertical center of the TREAT core. The first of these capsules (SETH-A) was unique with thermal insulation surrounding the rodlet. The SETH-B through -E rodlets were not surrounded by thermal insulation but were instead surrounded by an aluminum cage designed to support instrumentation. The SETH experiments were carried out to support research exploring a new calorimetry approach; this method requires taking temperature measurements of the rodlet's cladding surfaces during transient irradiation. The first transients were critical to understand the detector array field of view and signal response over long transients at low energies depositions. SETH-B2 and C had a reactivity addition of $1.15 \% \Delta \mathrm{k} / \mathrm{k}$ and allowed for a demonstration of the FMMS detector array behavior under increased reactor energy. The final two experiments, SETH-D and SETH-E, were aimed at reaching cladding melting temperatures with reactor energies of approximately $500 \mathrm{MJ}$ and $656 \mathrm{MJ}$. These final two tests served to demonstrate the FMMS's ability to track fuel motion during transient experiments at TREAT.

Key observations and conclusions:

- The FMMS' 96 detectors performed as expected during the SETH-A through -E experiments.

- Operational testing demonstrated that the FMMS platform can be effectively repositioned to re-center the detector array and allow its field of view to fully overserve fuel within test devices.

- This series of tests illustrated the importance of correctly choosing the lead absorber thickness in the FMMS system. The FMMS lead shielding was fully inserted, for a total $15.2 \mathrm{~cm}$, for the first five SETH experiments. It was reduced to $1.25 \mathrm{~cm}$ for SETH-D and -E. This thinner lead shielding led to saturation of the detectors during the peak of the reactor transient but at the lower reactor power there was higher sensitivity, which was important for capturing the expected fuel motion during the final two transients.

- During the SETH-D experiment the transient was clipped at approximately 3 seconds which was too early for the FMMS to capture rodlet motion. SETH-E had the same reactivity addition as SETH-D but the transient time was extended to 18 seconds. The FMMS captured the downward motion of the rodlet in this transient as the count rate significantly changed for the detectors viewing the bottom of the rodlet and the space immediately below the rodlet. FMMS snapshots throughout the transient helped identify the approximate time at which the motion initiated. Analysis of the detector data captured on a per-millisecond basis indicate the onset of downward motion started at $2.84 \pm 0.02$ seconds. 


\section{REFERENCES}

[1] N. Woolstenhulme, C. Baker, J. Bess, D. Chapman, C. Jensen, C. Hill, D. Wachs, S. Wilson, "Status Report for NEET In-Pile Sensor Irradiation Capabilities at TREAT," 2017. Web.

DOI:10.2172/1472112.

[2] "Separate Effects and Science-Based Irradiations (The MARCH System)." Transient Testing - Separate Effects and Science-Based Irradiations (The MARCH System), INL, https://transient.inl.gov/SitePages/Separate Effects and Science-Based Irradiations (The MARCH System).aspx.

[3] N. Woolstenhulme, A. Fleming, T. Holschuh, C. Jensen, D. Kamerman, D. Wachs, "Core-toSpecimen Energy Coupling Results of the First Modern Fueled Experiments in TREAT," 2019. INL/JOU-19-53086.

[4] J. D. Hix, D. L. Chichester, S. M. Watson, J. T. Johnson and S. J. Thompson, "Evaluation, Refurbishment, and Characterization of Fast Neutron Scintillators for the TREAT Fuel Motion Monitoring System," 2017 IEEE Nuclear Science Symposium and Medical Imaging Conference (NSS/MIC), Atlanta, GA, 2017, pp. 1-4.

[5] B.K. Heath, "TREAT FMMS Motion Control System Software Design,” TFR-993, 2019.

[6] N. Woolstenhulme, C. Baker, C. Jensen, D. Chapman, D. Imholte, N. Oldham, C. Hill, S. Snow, "Development of Irradiation Test Devices for Transient Testing," Nuclear Technology, 2019, pp.1-15. DOI: 10.1080/00295450.2019.1590072

[7] C. Race, "Experiments Operated in MARCH-BUSTER,” ESVC-SETH-A, 2018. 